\title{
Ground state and multiple solutions for a critical exponent problem
}

\author{
Z. Chen, N. Shioji, and W. Zou
}

\begin{abstract}
We study the following Brezis-Nirenberg type critical exponent equation which is related to the Yamabe problem:

$$
-\Delta u=\lambda u+|u|^{2^{*}-2} u, \quad u \in H_{0}^{1}(\Omega),
$$

where $\Omega$ is a smooth bounded domain in $\mathbb{R}^{N}(N \geq 3)$ and $2^{*}$ is the critical Sobolev exponent. We show that, if $N \geq 5$, this problem has at least $\left\lceil\frac{N+1}{2}\right\rceil$ pairs of nontrivial solutions for each fixed $\lambda \geq \lambda_{1}$, where $\lambda_{1}$ is the first eigenvalue of $-\Delta$ with the Dirichlet boundary condition. For $N \geq 3$, we give energy estimates from below for ground state solutions.
\end{abstract}

Mathematics Subject Classification (2010). 35J20, 35J25, 35J60.

\section{Introduction}

We study the following Brezis-Nirenberg critical Sobolev exponent problem:

$$
-\Delta u=\lambda u+|u|^{2^{*}-2} u, \quad u \in H_{0}^{1}(\Omega),
$$

where $\Omega$ is a smooth bounded domain of $\mathbb{R}^{N}(N \geq 3), 2^{*}:=\frac{2 N}{N-2}$ is the critical Sobolev exponent and $\lambda>0$. It is well known that solutions of problem (1.1) are critical points of the $C^{2}$ functional $I: H_{0}^{1}(\Omega) \rightarrow \mathbb{R}$ given by

$$
I(u)=\frac{1}{2} \int_{\Omega}\left(|\nabla u|^{2}-\lambda u^{2}\right) d x-\frac{1}{2^{*}} \int_{\Omega}|u|^{2^{*}} d x .
$$

Equation (1.1) arises in a geometric context in the Yamabe problem, whether a given metric $\mathcal{D}$ on a manifold $\mathcal{M}$ with scalar curvature $\mu$ can be conformally

Z. Chen and W. Zou are partially supported by NSFC (10871109); N. Shioji is partially supported by the Grant-in-Aid for Scientific Research (C) (No. 21540214) from Japan Society for the Promotion of Science. 
deformed to a metric $\mathcal{D}_{0}$ of constant scalar curvature. Let $\mathcal{D}_{0}=u^{4 /(N-2)} \mathcal{D}$, where $u$ is the conformal factor, the scalar curvature $\mu_{0}$ is given by the equation

$$
-\frac{4(N-1)}{N-2} \Delta_{\mathcal{M}} u+\mu u=\mu_{0} u|u|^{2^{*}-2},
$$

where $\Delta_{\mathcal{M}}$ is the Laplace-Beltrami operator on the manifold $\mathcal{M}$ with respect to the metric $\mathcal{D}[25,30]$. Let $\lambda_{n}$ be the $n$-th Dirichlet eigenvalue of $-\Delta$ on $\Omega$ counted with multiplicity. The pioneering paper on the Eq. (1.1) was due to Brezis and Nirenberg [6] in 1983 where the authors showed that for $N \geq 4$ and $\lambda \in\left(0, \lambda_{1}\right)$ problem (1.1) has at least one positive solution. The same conclusion was proved in [6] for $N=3$ when $\Omega$ is a ball and $\lambda \in\left(\lambda_{1} / 4, \lambda_{1}\right)$. By using a version of Pohozaev Identity, Eq. (1.1) has no nontrivial solution when $\lambda \leq 0$ and $\Omega$ is star-shaped. Since 1983, there has been a considerable number of papers on problem (1.1). Let us now briefly recall the main results obtained before. If $N \geq 4$ and $\lambda \neq \lambda_{n}$ for every $n \geq 1$, Capozzi et al. [7] showed that (1.1) has a nontrivial solution [31]. If $N \geq 5$ the same conclusion is true for every $\lambda>0[7,31]$. Arioli et al. [3] showed that problem (1.1) has only trivial radial solutions when $N=4, \Omega$ is the unit ball and $\lambda=\lambda_{1}$.

The first multiplicity result was obtained by Cerami et al. [8]. They proved that the number of solutions of (1.1) is bounded below by the number of eigenvalues of $(-\Delta, \Omega)$ lying in the open interval $\left(\lambda, \lambda+S|\Omega|^{-2 / N}\right)$, where $S$ is the best constant for the Sobolev embedding $D^{1,2}\left(\mathbb{R}^{N}\right) \hookrightarrow L^{2^{*}}\left(\mathbb{R}^{N}\right)$ and $|\Omega|$ is the Lebesgue measure of $\Omega$. If $N \geq 4$ and $\Omega$ is a ball, then for any $\lambda>0$, problem (1.1) has infinitely many sign-changing solutions which are built by using particular symmetry of the domain $\Omega$ [15]. If $N \geq 7$ and $\Omega$ is a ball, then for each $\lambda>0$, problem (1.1) has infinitely many sign-changing radial solutions [26]. Cerami et al. [10] proved for $N \geq 6$, problem (1.1) has two pairs of solutions on any smooth bounded domain. When $4 \leq N \leq 6$ and $\Omega$ is a ball, there is a $\lambda^{*}>0$ such that (1.1) has no radial solutions which change sign if $\lambda \in\left(0, \lambda^{*}\right)$ [2]. Devillanova and Solimini [12] showed that, if $N \geq 7$, problem (1.1) has infinitely many solutions for each $\lambda>0$. There is no information on the sign-changingness of the solutions obtained in [12]. Recently, Schechter and Zou [23] proved that, if $N \geq 7$, problem (1.1) has infinitely many signchanging solutions for each $\lambda>0$. In 2005, Clapp and Weth [11] got finitely many solutions to (1.1) for each $\lambda>0$ and $N \geq 4$. The main result of [11] says that if $0<\lambda<\lambda_{1}$, then (1.1) has at least $\left\lceil\frac{N+2}{2}\right\rceil$ pairs of nontrivial solutions; if $\lambda_{n}<\lambda<\lambda_{n+1}$, then (1.1) has at least $\left\lceil\frac{N+1}{2}\right\rceil$ pairs of nontrivial solutions; if $\lambda=\lambda_{n+1}=\cdots=\lambda_{n+m}$ is an eigenvalue of multiplicity $m<N+2$, then (1.1) has at least $\left\lceil\frac{N+1-m}{2}\right\rceil$ pairs of nontrivial solutions. All these solutions satisfy $I(u)<\frac{2}{N} S^{N / 2}$. Here, $\lceil a\rceil$ is the least integer $n$ satisfying $n \geq a$ for each $a>0$.

From the results of [11] we see that if $\lambda$ is an eigenvalue of multiplicity $m \geq N+2$, there is no information about the existence and multiplicity of solutions to (1.1) for $N<7$. In this paper, we give a partial answer to this question. Precisely, we shall prove the following theorem.

Theorem 1.1. Let $N \geq 5$ and $\lambda \geq \lambda_{1}$, then (1.1) has at least $\left\lceil\frac{N+1}{2}\right\rceil$ pairs of nontrivial solutions. These solutions satisfy $I(u)<\frac{2}{N} S^{N / 2}$. 
Here we will use a constraint method and the usual Krasnoselskii genus theory without $(P S)_{c}$ type condition to prove Theorem 1.1, and we do not need the capacity or the equivariant relative category that are used in [11].

Another natural question is about the existence and energy estimates of ground state solutions to problem (1.1). The ground state refers to minimizers of the corresponding energy within the set of nontrivial solutions. When $N \geq 4$ and $0<\lambda<\lambda_{1}$, the existence of (positive) ground state solutions was proved by Brezis and Nirenberg [6]. Very recently, Szulkin, Weth and Willem [22] studied the case $\lambda \geq \lambda_{1}$. They proved that, for $N=4$ and $\lambda_{n}<\lambda<\lambda_{n+1}$ or $N \geq 5$ and $\lambda_{n} \leq \lambda<\lambda_{n+1}(n \geq 1)$, problem (1.1) has ground state solutions. On the other hand, if $0<\lambda<\lambda_{1}$, we know that if $u$ is a solution of problem (1.1), then

$$
I(u)>\frac{1}{N}\left(1-\frac{\lambda}{\lambda_{1}}\right)^{N / 2} S^{N / 2} .
$$

In fact, by $I^{\prime}(u) u=0$ we get

$$
\begin{aligned}
\left(1-\frac{\lambda}{\lambda_{1}}\right) \int_{\Omega}|\nabla u|^{2} d x & \leq \int_{\Omega}\left(|\nabla u|^{2}-\lambda u^{2}\right) d x \\
& =\int_{\Omega}|u|^{2^{*}} d x<\left(\frac{1}{S} \int_{\Omega}|\nabla u|^{2} d x\right)^{2^{*} / 2} .
\end{aligned}
$$

So

$$
\int_{\Omega}|\nabla u|^{2} d x>\left(1-\frac{\lambda}{\lambda_{1}}\right)^{(N-2) / 2} S^{N / 2}
$$

and we get

$$
I(u)>\frac{1}{N}\left(1-\frac{\lambda}{\lambda_{1}}\right)^{N / 2} S^{N / 2} .
$$

Therefore, if $u$ is a ground state solution, then the ground state energy satisfies $I(u)>\frac{1}{N}\left(1-\frac{\lambda}{\lambda_{1}}\right)^{N / 2} S^{N / 2}$. However, including [22], there is no similar estimate of energy to the ground state solutions for the case $\lambda \geq \lambda_{1}$. Here we give a positive answer to this open question. Precisely, we shall prove the following theorem.

Theorem 1.2. Let $\lambda_{n} \leq \lambda<\lambda_{n+1}$ for some $n \geq 1$.

(1) If $N \geq 5$, then problem (1.1) has a ground state solution $u$.

(2) If $N=4$ and $\lambda_{n}<\lambda<\lambda_{n+1}$, then problem (1.1) has a ground state solution $u$.

(3) If $N=3$ and $\lambda_{n+1}-S|\Omega|^{-2 / N}<\lambda<\lambda_{n+1}$, then problem (1.1) has a ground state solution $u$.

These solutions satisfy $I(u)>\frac{1}{N}\left(1-\frac{\lambda}{\lambda_{n+1}}\right)^{N / 2} S^{N / 2}$.

As has been pointed out above, the existence of ground state solutions in case $N \geq 4$ in Theorem 1.2 are not new, and can be found in [22]. They gave a minimax characterization of the ground states and used the Nehari manifold introduced by Pankov [19] to prove their results. Here we will give a direct 
proof which is much simpler than the proof of [22]. Moreover, we can get an energy estimate as stated in Theorem 1.2. In case $N=3$, one nontrivial solution had been found in [8], where whether the solution is a ground state had not been determined. The novelty of the current paper is that we can prove there exist ground state solutions and get the energy estimates.

We also consider the following critical biharmonic problem under the Dirichlet boundary condition

$$
\Delta^{2} u-\lambda u=|u|^{\frac{8}{N-4}} u \quad \text { in } \Omega, \quad u=|\nabla u|=0 \quad \text { on } \partial \Omega
$$

and the one under the Navier boundary condition

$$
\Delta^{2} u-\lambda u=|u|^{\frac{8}{N-4}} u \quad \text { in } \Omega, \quad u=\Delta u=0 \quad \text { on } \partial \Omega .
$$

These kind of problems have received much interest in recent years, and were studied by many authors, see $[4,13,16-18,20,28,29]$. In particular, Clapp and Weth [11] obtained the following result in case $N \geq 8$ : if $\lambda>0$ is not a Dirichlet (respectively Navier) eigenvalue of $\Delta^{2}$ on $\Omega$, then problem (1.2) (respectively (1.3)) has at least $\lceil(N+1) / 2\rceil$ pairs of nontrivial solutions; if $\lambda$ is an eigenvalue of multiplicity $m<N+2$, then it has at least $\lceil(N+1-m) / 2\rceil$ pairs of nontrivial solutions.

From the results of [11] we see that if $\lambda$ is an eigenvalue of multiplicity $m \geq N+2$, there is no information about the multiplicity of solutions to (1.2) (respectively (1.3)). Here we give a partial answer to this question. Let $\widetilde{\lambda}_{n}$ be the $n$-th eigenvalue of $\Delta^{2}$ with boundary condition (1.2) (respectively (1.3)) on $\Omega$ counted with multiplicity. We shall prove the following theorem.

Theorem 1.3. Let $N \geq 10$ and $\lambda \geq \widetilde{\lambda}_{1}$, then problem (1.2) (respectively (1.3)) has at least $\lceil(N+1) / 2\rceil$ pairs of nontrivial solutions.

We can also study the ground state solutions to problem (1.2) and (1.3). To the best of our knowledge, it seems there is no information about the existence and energy estimates of the ground state solutions to problem (1.2) and (1.3) in case $\lambda \geq \widetilde{\lambda}_{1}$. Here we will give the following theorem.

Theorem 1.4. Let $\widetilde{\lambda}_{n} \leq \lambda<\widetilde{\lambda}_{n+1}$ for some $n \geq 1$.

(1) If $N \geq 10$, then problem (1.2) (respectively (1.3)) has a ground state solution $u$.

(2) If $N=8,9$ and $\lambda \neq \widetilde{\lambda}_{n}$, then problem (1.2) (respectively (1.3)) has a ground state solution $u$.

These solutions satisfy $\tilde{I}(u)>\frac{2}{N}\left(1-\lambda / \widetilde{\lambda}_{n+1}\right)^{N / 4} \widetilde{S}^{N / 4}$, where $\tilde{I}$ is the energy functional of (1.2) (respectively (1.3)) defined in (4.1).

The existence of nontrivial solutions in case $N \geq 8$ in Theorem 1.4 are not new, and can be seen in [16]. Here we will prove there exist ground state solutions and get the energy estimates. Since methods in the proofs of Theorem 1.3 and 1.4 are similar to Theorems 1.1 and 1.2, we will give sketch proofs of Theorem 1.3 and 1.4 in Sect. 4. 


\section{Proof of Theorem 1.1}

In this section we assume that $N \geq 5$ and $\lambda_{n} \leq \lambda<\lambda_{n+1}$ for some $n \geq 1$. Throughout this paper, we denote the norm of $L^{p}(\Omega)$ by $\|u\|_{p}=\left(\int_{\Omega}|u|^{p} d x\right)^{\frac{1}{p}}$, and positive constants (possibly different) by $C$. Let $H:=H_{0}^{1}(\Omega)$ be the usual Sobolev space with the inner product $(u, v)=\int_{\Omega} \nabla u \nabla v d x$ and the corresponding norm $\|u\|=(u, u)^{\frac{1}{2}}$. We choose a sequence of $L^{2}$-normalized orthonormal eigenfunctions $e_{k}$ corresponding to Dirichlet eigenvalues $\lambda_{k}, k \in \mathbb{N}$, that is $-\Delta e_{k}=\lambda_{k} e_{k}$ as $x \in \Omega$ and $e_{k}=0$ when $x \in \partial \Omega$. Then $e_{k} \in C^{\infty}(\Omega)$ and there is a constant $C_{0}>0$ such that $\left|e_{k}(x)\right| \leq C_{0}$ for $x \in \Omega$. We set

$$
V^{-}:=\operatorname{span}\left\{e_{1}, \ldots, e_{n}\right\}, \quad V^{+}:=\overline{\operatorname{span}\left\{e_{j}: j \geq n+1\right\}}
$$

As pointed out above, the solutions of problem (1.1) correspond to critical points of the $C^{2}$ functional $I: H \rightarrow \mathbb{R}$ given by

$$
I(u)=\frac{1}{2} \int_{\Omega}\left(|\nabla u|^{2}-\lambda u^{2}\right) d x-\frac{1}{2^{*}} \int_{\Omega}|u|^{2^{*}} d x .
$$

As usual, we say that a sequence $\left(u_{m}\right)$ in $H$ is a $(P S)_{c}$ sequence for $I$ if

$$
I\left(u_{m}\right) \rightarrow c, \quad\left\|I^{\prime}\left(u_{m}\right)\right\| \rightarrow 0, \quad \text { as } \quad m \rightarrow \infty .
$$

As in [25], we consider a new functional

$$
J(u):=\frac{\int_{\Omega}\left(|\nabla u|^{2}-\lambda u^{2}\right) d x}{\left(\int_{\Omega}|u|^{2^{*}} d x\right)^{2 / 2^{*}}}=\int_{\Omega}\left(|\nabla u|^{2}-\lambda u^{2}\right) d x
$$

defined on $M:=\left\{u \in H:\|u\|_{2^{*}}=1\right\}$. Then $M \subset H$ is a complete Hilbert manifold, invariant under the involution $u \rightarrow-u$. Moreover, we have that $J \in C^{1}(M, \mathbb{R})$, and $u \in M$ is a critical point of $J$ with $J(u)=\beta>0$, if and only if $\tilde{u}:=\beta^{\frac{1}{2^{*}-2}} u$ is a critical point of $I$ with $I(\tilde{u})=\frac{1}{N} \beta^{N / 2}>0$. Clearly, $\left(u_{m}\right)$ is a $(P S)_{\beta}$ sequence for $J$ if and only if the sequence $\left(\tilde{u}_{m}\right)$, where $\tilde{u}_{m}:=\beta^{\frac{1}{2^{*}-2}} u_{m}$, is a $(P S)_{\tilde{\beta}}$ sequence for $I$ with $\tilde{\beta}=\frac{1}{N} \beta^{N / 2}$. Here, we say that a sequence $\left(u_{m}\right)$ in $M$ is a $(P S)_{\beta}$ sequence for $J$ if

$$
J\left(u_{m}\right) \rightarrow \beta, \quad\left\|J^{\prime}\left(u_{m}\right)\right\| \rightarrow 0, \quad \text { as } \quad m \rightarrow \infty .
$$

Denote

$$
\begin{aligned}
\hat{M} & :=\left\{u \in M: J^{\prime}(u) \neq 0\right\}, \quad J^{\beta}:=\{u \in M: J(u) \leq \beta\}, \\
K^{\beta} & :=\left\{u \in M: J^{\prime}(u)=0, J(u)=\beta\right\} .
\end{aligned}
$$

Note that $J(u)=J(-u)$. It is well known [25] that there is an odd pseudogradient vector field, i.e., there exists an odd Lipschitz continuous map $\nu: \hat{M} \rightarrow T M$ with $\nu(u) \in T_{u} M$ and $\left.\|\nu(u)\|<2\left\|J^{\prime}(u)\right\|,\left\langle J^{\prime}(u), \nu(u)\right\rangle\right\rangle$ $\left\|J^{\prime}(u)\right\|^{2}$. We have the following deformation lemma on the Hilbert manifold $M$ without $(P S)$ condition.

Lemma 2.1. Let $\varepsilon, \delta>0, \beta \in \mathbb{R}$ and $D \subset M$ be a symmetric subset (i.e., $D=-D)$ such that $\left\|J^{\prime}(u)\right\| \geq \frac{4 \varepsilon}{\delta}$ for $u \in J^{-1}[\beta-2 \varepsilon, \beta+2 \varepsilon] \cap D_{2 \delta}$, where $D_{\delta}:=\{u \in M: \operatorname{dist}(u, D) \leq \delta\}$. Then there exists a map $\eta \in C^{1}([0,1] \times M, M)$ such that $\eta(t, \cdot): M \rightarrow M$ is an odd homeomorphism for any $t \in[0,1]$ and 
(1) $\eta(0, u)=u, \quad \forall u \in M$;

(2) $\eta(t, u)=u, \quad \forall u \notin J^{-1}[\beta-2 \varepsilon, \beta+2 \varepsilon] \cap D_{2 \delta}$;

(3) $\eta\left(1, J^{\beta+\varepsilon} \cap D\right) \subset J^{\beta-\varepsilon}$.

Proof. Let $A:=J^{-1}[\beta-2 \varepsilon, \beta+2 \varepsilon] \cap D_{2 \delta}, \quad B:=J^{-1}[\beta-\varepsilon, \beta+\varepsilon] \cap D_{\delta}$ and define $\rho: M \rightarrow \mathbb{R}$ by

$$
\rho(u)=\frac{\operatorname{dist}(u, M \backslash A)}{\operatorname{dist}(u, M \backslash A)+\operatorname{dist}(u, B)} .
$$

Let

$$
f(u)= \begin{cases}-\rho(u) \frac{\nu(u)}{\|\nu(u)\|} & \text { if } u \in \hat{M}, \\ 0 & \text { if } u \in M \backslash \hat{M},\end{cases}
$$

and consider the Cauchy problem $\frac{d}{d t} \omega=f(\omega)$ with $\omega(0, u)=u$. Then this problem has a unique solution $\omega(t, u)$ for $t \in \mathbb{R}$ such that $\omega(t, \cdot)$ is an odd homeomorphism with respect to $u \in M$. Let $\eta(t, u)=\omega(\delta t, u)$. It is easy to see that (1) and (2) holds. Note that

$$
\frac{d}{d t} J(\eta(t, u))=\frac{d}{d t} J(\omega(\delta t, u))=-\delta\left\langle J^{\prime}(\eta), \rho(\eta) \frac{\nu(\eta)}{\|\nu(\eta)\|}\right\rangle \leq-\delta \rho(\eta) \frac{\left\|J^{\prime}(\eta)\right\|^{2}}{\|\nu(\eta)\|},
$$

we get that $J(\eta(\cdot, u))$ is non-increasing with respect to $t$. For any $u \in J^{\beta+\varepsilon} \cap D$,

$$
\|\eta(t, u)-u\|=\left\|\int_{0}^{t} \frac{d}{d s} \eta(s, u) d s\right\| \leq \int_{0}^{t} \delta\|f(w)\| d s \leq \delta t,
$$

we get that $\eta(t, u) \in D_{\delta}, \forall t \in[0,1]$. If there exists $t_{0} \in[0,1)$ such that $J\left(\eta\left(t_{0}, u\right)\right) \leq \beta-\varepsilon$, then $J(\eta(1, u)) \leq J\left(\eta\left(t_{0}, u\right)\right) \leq \beta-\varepsilon$, that is $\eta(1, u) \in J^{\beta-\varepsilon}$. If $J(\eta(t, u))>\beta-\varepsilon$ for all $t \in[0,1)$, we have that $\eta(t, u) \in B$. Hence, $\rho(\eta(t, u)) \equiv 1$. It follows that

$$
\begin{aligned}
J(\eta(1, u)) & =J(u)-\delta \int_{0}^{1}\left\langle J^{\prime}(\eta), \frac{\nu(\eta)}{\|\nu(\eta)\|}\right\rangle d t \\
& \leq J(u)-\delta \int_{0}^{1} \frac{\left\|J^{\prime}(\eta)\right\|}{2} d t \leq \beta-\varepsilon,
\end{aligned}
$$

that means $\eta(1, u) \in J^{\beta-\varepsilon}$. Therefore, (3) holds.

Now, for $j \in \mathbb{N}, j \geq n+1$, we define $\Sigma_{j}:=\{A \subset M: A=-A=\bar{A}$, $\gamma(A) \geq j\}$, where $\gamma$ denotes the usual Krasnoselskii genus, and consider

$$
\beta_{j}:=\inf _{A \in \Sigma_{j}} \sup _{u \in A} J(u) .
$$

Note that $j \geq n+1$, we have $A \cap\left\{u \in V^{+}:\|u\|_{2^{*}}=1\right\} \neq \emptyset$ for any $A \in \Sigma_{j}$, since $\gamma(A)>n$. Therefore $\beta_{j}>0$ for all $j \geq n+1$.

Lemma 2.2. For $j \geq n+1$, there exists a $(P S)_{\beta_{j}}$ sequence $\left(u_{m}\right)$ for $J$.

Proof. Fix $j \geq n+1$. If there is no $(P S)_{\beta_{j}}$ sequence $\left(u_{m}\right)$ for $J$, then there exists $\varepsilon>0$ such that $\left\|J^{\prime}(u)\right\| \geq 4 \varepsilon$ for $u \in J^{-1}\left[\beta_{j}-2 \varepsilon, \beta_{j}+2 \varepsilon\right]$. Let $D=M, \delta=1$. Then by Lemma 2.1 there is an continuous map $\eta \in C^{1}([0,1] \times M, M)$ with $\eta\left(1, J^{\beta_{j}+\varepsilon}\right) \subset J^{\beta_{j}-\varepsilon}$. There exists an $A \in \Sigma_{j}$ such that sup $J(u)<\beta_{j}+\varepsilon$. Since 
$\eta(1, \cdot)$ is odd, $\gamma(\eta(1, A)) \geq \gamma(A) \geq j$, that is $\eta(1, A) \in \Sigma_{j}$. While $\eta(1, A) \subset$ $\eta\left(1, J^{\beta_{j}+\varepsilon}\right) \subset J^{\beta_{j}-\varepsilon}$, which is a contradiction with the definition of $\beta_{j}$.

For $\varepsilon>0$ and $y \in \mathbb{R}^{N}$, we consider the Aubin-Talenti instanton $[1,27]$ $U_{\varepsilon, y} \in D^{1,2}\left(\mathbb{R}^{N}\right)$ defined by

$$
U_{\varepsilon, y}:=[N(N-2)]^{\frac{N-2}{4}}\left(\frac{\varepsilon}{\varepsilon^{2}+|x-y|^{2}}\right)^{\frac{N-2}{2}}=A_{0}\left(\frac{\varepsilon}{\varepsilon^{2}+|x-y|^{2}}\right)^{\frac{N-2}{2}}
$$

where $A_{0}:=[N(N-2)]^{\frac{N-2}{4}}$ is a constant. Then $U_{\varepsilon, y}$ satisfies the equation $-\Delta u=|u|^{2^{*}-2} u$ on $\mathbb{R}^{N}$ and $\int_{\mathbb{R}^{N}}\left|\nabla U_{\varepsilon, y}\right|^{2} d x=\int_{\mathbb{R}^{N}}\left|U_{\varepsilon, y}\right|^{2^{*}} d x=S^{N / 2}$. Let

$$
E:=\left\{U_{\varepsilon, y}: \varepsilon>0, y \in \mathbb{R}^{N}\right\}
$$

Then $E$ contains all positive solutions of the equation $-\Delta u=|u|^{2^{*}-2} u$ on $\mathbb{R}^{N}$.

Lemma 2.3. Let $\left(u_{m}\right)$ be a $(P S)_{\beta_{j}}$ sequence for $J$. Up to a subsequence, the following properties hold.

(a) If $0<\beta_{j}<S$, then $\left(u_{m}\right)$ converges in $M$ and $\beta_{j}$ is a critical value of $J$.

(b) If $S<\beta_{j}<2^{2 / N} S$, then one of the following conclusions holds:

(b.1) $\quad\left(u_{m}\right)$ converges in $M$, that is $\beta_{j}$ is a critical value of $J$.

(b.2) There is a critical point $u$ of $J$ with $J(u)=\beta_{*}=\left(\beta_{j}^{N / 2}-S^{N / 2}\right)^{2 / N} \in$ $(0, S)$ such that

$\operatorname{dist}\left(\beta_{j}^{\frac{1}{2^{*}-2}} u_{m}-\beta_{*^{\frac{1}{2^{*}-2}}} u, E\right) \rightarrow 0$ or $\operatorname{dist}\left(\beta_{j}^{\frac{1}{2^{*}-2}} u_{m}-\beta_{*^{\frac{1}{2^{*}-2}}} u,-E\right) \rightarrow 0$.

(c) If $\beta_{j}=S$, then one of the following conclusions holds:

(c.1) $\left(u_{m}\right)$ converges in $M$ and $\beta_{j}$ is a critical value of $J$.

(c.2) $\operatorname{dist}\left(\beta_{j}^{\frac{1}{2^{*}-2}} u_{m}, E\right) \rightarrow 0$ or $\operatorname{dist}\left(\beta_{j}^{\frac{1}{2^{*}-2}} u_{m},-E\right) \rightarrow 0$.

Proof. This lemma is inspired by a similar lemma for the functional $I$ (see Lemma 7 in [11]), and we give a sketch proof. Since $\left(u_{m}\right)$ is a $(P S)_{\beta_{j}}$ sequence for $J$, it follows that sequence $\left(\tilde{u}_{m}\right)$, where $\tilde{u}_{m}:=\beta_{j}^{\frac{1}{2^{*}-2}} u_{m}$, is a $(P S)_{\tilde{\beta}_{j}}$ sequence for $I$ with $\tilde{\beta}_{j}=\frac{1}{N} \beta_{j}^{N / 2}$. By Struwe's global compactness result for $I[24,25]$, up to a subsequence, if $0<\tilde{\beta}_{j}<\frac{1}{N} S^{N / 2}$, it follows that $\tilde{\beta}_{j}$ is a critical value of $I$, so $(a)$ holds; if $\frac{1}{N} S^{N / 2}<\tilde{\beta}_{j}<\frac{2}{N} S^{N / 2}$, then $\tilde{\beta}_{j}$ is a critical value of $I$, which responds to $(b .1)$. Otherwise, there is a critical point $\tilde{u}$ of $I$ with $I(\tilde{u})=\tilde{\beta}_{j}-\frac{1}{N} S^{N / 2}=\frac{1}{N} \beta_{*}^{N / 2}$ such that either $\operatorname{dist}\left(\tilde{u}_{m}-\tilde{u}, E\right) \rightarrow 0$ or $\operatorname{dist}\left(\tilde{u}_{m}-\tilde{u},-E\right) \rightarrow 0$, which reduces to $(b .2)$. If $\tilde{\beta}_{j}=\frac{1}{N} S^{N / 2}$, then $\tilde{\beta}_{j}$ is a critical value of $I$ and (c.1) follows. If not, we then have either $\operatorname{dist}\left(\tilde{u}_{m}, E\right) \rightarrow 0$ or $\operatorname{dist}\left(\tilde{u}_{m},-E\right) \rightarrow 0$ and we $\operatorname{get}(c .2)$.

Lemma 2.4. If $\beta_{j}=\beta_{j+1}<2^{2 / N} S$, then $K^{\beta_{j}}$ is infinite.

Proof. Let $\beta=\beta_{j}=\beta_{j+1}$. If $0<\beta<S$, then the $(P S)_{\beta}$ condition holds from Lemma $2.3(a)$, then by a standard argument we get $\gamma\left(K^{\beta}\right) \geq 2$, that is, $K^{\beta}$ is 
infinite. If $S<\beta<2^{2 / N} S$, we denote $\beta_{*}:=\left(\beta^{N / 2}-S^{N / 2}\right)^{2 / N}>0$ and define

$$
\begin{aligned}
W_{+}(\delta) & :=\left\{v \in M: \operatorname{dist}\left(\beta^{\frac{1}{2^{*}-2}} v-\beta_{*}^{\frac{1}{2^{*}-2}} u, E\right) \leq \delta \text { for some } u \in K^{\beta_{*}}\right\}, \\
W_{-}(\delta) & :=\left\{v \in M: \operatorname{dist}\left(\beta^{\frac{1}{2^{*}-2}} v-\beta_{*}^{\frac{1}{2^{*}-2}} u,-E\right) \leq \delta \text { for some } u \in K^{\beta_{*}}\right\}, \\
W(\delta) & :=W_{+}(\delta) \cup W_{-}(\delta),
\end{aligned}
$$

where $\delta>0$. By a similar proof as in [11], we get that $W_{+}(\delta) \cap W_{-}(\delta)=\emptyset$ for $\delta>0$ small enough. If $K^{\beta}$ is finite, then $\gamma\left(K^{\beta}\right) \leq 1$. Fix $\delta^{\prime}>0$ small enough such that $W_{+}\left(\delta^{\prime}\right) \cap W_{-}\left(\delta^{\prime}\right)=\emptyset, \gamma\left(B_{\delta^{\prime}}\left(K^{\beta}\right)\right)=\gamma\left(K^{\beta}\right)$ and $B_{\delta^{\prime}}\left(K^{\beta}\right) \cap W\left(\delta^{\prime}\right)=\emptyset$, where $B_{\delta^{\prime}}\left(K^{\beta}\right)=\left\{u \in M: \operatorname{dist}\left(u, K^{\beta}\right) \leq \delta^{\prime}\right\}$. Then $\gamma\left(\left(B_{\delta^{\prime}}\left(K^{\beta}\right) \cup W\left(\delta^{\prime}\right)\right) \leq 1\right.$. By Lemma 2.3, there is an $\varepsilon>0$ such that

$$
\left\|J^{\prime}(u)\right\| \geq \frac{16 \varepsilon}{\delta^{\prime}} \quad \text { for } u \in J^{-1}[\beta-2 \varepsilon, \beta+2 \varepsilon] \backslash \operatorname{int}\left(B_{\delta^{\prime} / 2}\left(K^{\beta}\right) \cup W\left(\delta^{\prime} / 2\right)\right) \text {. }
$$

Let $D:=H \backslash\left(B_{\delta^{\prime}}\left(K^{\beta}\right) \cup W\left(\delta^{\prime}\right)\right)$ and $\delta=\delta^{\prime} / 4$. Then by Lemma 2.1, there is an odd continuous map $\eta \in C^{1}([0,1] \times M, M)$ such that

$$
\eta\left(1, J^{\beta+\varepsilon} \backslash\left(B_{\delta^{\prime}}\left(K^{\beta}\right) \cup W\left(\delta^{\prime}\right)\right)\right) \subset J^{\beta-\varepsilon} .
$$

Thus

$$
\begin{aligned}
j+1 & \leq \gamma\left(J^{\beta+\varepsilon}\right) \leq \gamma\left(J^{\beta+\varepsilon} \backslash\left(B_{\delta^{\prime}}\left(K^{\beta}\right) \cup W\left(\delta^{\prime}\right)\right)\right)+\gamma\left(B_{\delta^{\prime}}\left(K^{\beta}\right) \cup W\left(\delta^{\prime}\right)\right) \\
& \leq \gamma\left(J^{\beta-\varepsilon}\right)+1 \leq j-1+1 \leq j,
\end{aligned}
$$

which is a contradiction. Hence, $K^{\beta}$ is infinite. If $\beta=S$, the proof is similar to the case $S<\beta<2^{2 / N} S$. We omit the details.

We set $B(x, r):=\left\{y \in \mathbb{R}^{N}:|y-x|<r\right\}$. Without loss of generality, we may assume that $0 \in \Omega$. Then we have $B\left(0, \frac{2}{m}\right) \in \Omega$ for $m$ large enough. Let $e_{i}^{m}:=\xi_{\frac{2}{m}} e_{i}$ and $V_{m}^{-}:=\operatorname{span}\left\{e_{1}^{m}, \ldots, e_{n}^{m}\right\}$, where

$$
\xi_{\eta}(x):= \begin{cases}0 & \text { if } x \in B\left(0, \frac{\eta}{2}\right), \\ \frac{2}{\eta}|x|-1 & \text { if } x \in B(0, \eta) \backslash B\left(0, \frac{\eta}{2}\right), \\ 1 & \text { if } x \in \Omega \backslash B(0, \eta),\end{cases}
$$

for any $\eta>0$. Then it follows that $\left|\nabla \xi_{\frac{2}{m}}(x)\right| \leq m$, and $e_{i}^{m} \in H_{0}^{1}\left(\Omega \backslash B\left(0, \frac{1}{m}\right)\right)$. We have the following lemma.

Lemma 2.5. (a) $\left\|e_{i}^{m}-e_{i}\right\| \rightarrow 0$ as $m \rightarrow+\infty$;

(b) There exists an $m_{0} \in \mathbb{N}$ such that for any $m \geq m_{0}$ it holds

$$
\max _{\left\{u \in V_{m}^{-}:\|u\|_{2}=1\right\}}\|u\|^{2} \leq \lambda_{n}+C_{1} m^{-N+2},
$$

where $C_{1}$ is a constant independent of $m$.

The proof of $(a)$ can be found in [14] and the proof of $(b)$ can be found in [9]. In fact they proved some similar conclusions with Hardy potential $\frac{\mu}{|x|^{2}}$ for $\mu \in\left[0,\left(\frac{N-2}{2}\right)^{2}\right)$. As a direct consequence of Lemma 2.5, we have the following lemma which is important for our proof. 
Lemma 2.6. For any $m \geq m_{0}$ it holds

$$
\sup _{u \in V_{m}^{-}} I(u) \leq C_{2} m^{-\frac{N(N-2)}{2}},
$$

where $C_{2}$ is a constant independent of $m$.

Proof. Note that $\lambda_{n} \leq \lambda$. For any $m \geq m_{0}$ and $u \in V_{m}^{-}$, by Holder inequality and $(b)$ of Lemma 2.5, we have

$$
\begin{aligned}
I(u) & \leq \frac{\lambda_{n}-\lambda}{2} \int_{\Omega} u^{2} d x+\frac{C_{1}}{2} m^{-N+2} \int_{\Omega} u^{2} d x-\frac{1}{2^{*}} \int_{\Omega}|u|^{2^{*}} d x \\
& \leq C m^{-N+2}\|u\|_{2^{*}}^{2}-\frac{1}{2^{*}}\|u\|_{2^{*}}^{2^{*}} \\
& \leq \max _{t \geq 0}\left(C m^{-N+2} t^{2}-\frac{1}{2^{*}} t^{2^{*}}\right) \\
& \leq C_{2} m^{-\frac{N(N-2)}{2}} .
\end{aligned}
$$

Thus, (2.3) holds.

Define $U_{\varepsilon}:=U_{\varepsilon, 0}=A_{0}\left(\frac{\varepsilon}{\varepsilon^{2}+|x|^{2}}\right)^{\frac{N-2}{2}}$ and let $r_{1}=\frac{1}{2 m}, r_{2}=\frac{r_{1}}{3}$. Then for any $r \in\left(0, r_{2}\right]=\left(0, \frac{1}{6 m}\right]$, we define a cut-off function of $U_{\varepsilon}$ by

$$
U_{\varepsilon}^{r}(x)= \begin{cases}U_{\varepsilon}(x)-A_{0}\left(\frac{\varepsilon}{\varepsilon^{2}+r^{2}}\right)^{\frac{N-2}{2}} & \text { if } x \in B(0, r), \\ 0 & \text { if } x \in \Omega \backslash B(0, r) .\end{cases}
$$

Similar cut-off definitions can be seen in [9, 14]. Then $U_{\varepsilon}^{r} \in H_{0}^{1}(B(0, r)) \subset$ $H_{0}^{1}(\Omega)$. Note $(2.2)$, and define $\xi_{\eta} \equiv 1$ for $\eta=0$. Let $0 \leq 2 \eta<\varepsilon<r$, then the following lemma holds.

Lemma 2.7. For any $y \in \Omega$, the following estimates hold.

$$
\begin{gathered}
\int_{\Omega}\left|\nabla\left(\xi_{\eta}(x-y) U_{\varepsilon}^{r}(x)\right)\right|^{2} d x \leq S^{N / 2}-C\left(\frac{\varepsilon}{r}\right)^{N-2}+C\left(\frac{\eta}{\varepsilon}\right)^{N-2}, \\
\int_{\Omega}\left|\xi_{\eta}(x-y) U_{\varepsilon}^{r}(x)\right|^{2^{*}} d x \geq S^{N / 2}-C\left(\frac{\varepsilon}{r}\right)^{N-2}-C\left(\frac{\eta}{\varepsilon}\right)^{N-2} .
\end{gathered}
$$

Proof. First, we assume $\eta=0$. Note $\nabla U_{\varepsilon}(x)=-(N-2) A_{0} \varepsilon^{\frac{N-2}{2}} \frac{x}{\left(\varepsilon^{2}+|x|^{2}\right)^{N / 2}}$, we have

$$
\begin{aligned}
\int_{\Omega}\left|\nabla U_{\varepsilon}^{r}\right|^{2} d x & =\int_{B(0, r)}\left|\nabla U_{\varepsilon}\right|^{2} d x=S^{N / 2}-\int_{\mathbb{R}^{N} \backslash B(0, r)}\left|\nabla U_{\varepsilon}\right|^{2} d x \\
& =S^{N / 2}-C \varepsilon^{N-2} \int_{\mathbb{R}^{N} \backslash B(0, r)} \frac{|x|^{2}}{\left(\varepsilon^{2}+|x|^{2}\right)^{N}} d x \\
& =S^{N / 2}-C \varepsilon^{N-2} \int_{r}^{+\infty} \frac{\rho^{N+1}}{\left(\varepsilon^{2}+\rho^{2}\right)^{N}} d \rho \\
& \leq S^{N / 2}-C\left(\frac{\varepsilon}{r}\right)^{N-2} .
\end{aligned}
$$


Hence, (2.4) holds.

$$
\begin{aligned}
\int_{\Omega}\left|U_{\varepsilon}^{r}\right|^{2^{*}} d x= & \int_{B(0, r)}\left|U_{\varepsilon}^{r}\right|^{2^{*}} d x \\
\geq & \int_{B(0, r)}\left|U_{\varepsilon}\right|^{2^{*}} d x-2^{*} \int_{B(0, r)}\left|U_{\varepsilon}^{r}\right|^{2^{*}-1} A_{0}\left(\frac{\varepsilon}{\varepsilon^{2}+r^{2}}\right)^{\frac{N-2}{2}} d x \\
= & \int_{\mathbb{R}^{N}}\left|U_{\varepsilon}\right|^{2^{*}} d x-\int_{\mathbb{R}^{N} \backslash B(0, r)}\left|U_{\varepsilon}\right|^{2^{*}} d x \\
& -2^{*} \int_{B(0, r)}\left|U_{\varepsilon}^{r}\right|^{2^{*}-1} A_{0}\left(\frac{\varepsilon}{\varepsilon^{2}+r^{2}}\right)^{\frac{N-2}{2}} d x
\end{aligned}
$$

While

$$
\int_{\mathbb{R}^{N} \backslash B(0, r)}\left|U_{\varepsilon}\right|^{2^{*}} d x=C \int_{r}^{+\infty}\left(\frac{\varepsilon}{\varepsilon^{2}+\rho^{2}}\right)^{N} \rho^{N-1} d \rho \leq C \varepsilon^{N} r^{-N}
$$

and

$$
\begin{aligned}
& 2^{*} \int_{B(0, r)}\left|U_{\varepsilon}^{r}\right|^{2^{*}-1} A_{0}\left(\frac{\varepsilon}{\varepsilon^{2}+r^{2}}\right)^{\frac{N-2}{2}} d x \\
& \quad=C \int_{0}^{r}\left(\frac{\varepsilon}{\varepsilon^{2}+\rho^{2}}\right)^{\frac{N+2}{2}}\left(\frac{\varepsilon}{\varepsilon^{2}+r^{2}}\right)^{\frac{N-2}{2}} \rho^{N-1} d \rho \\
& \quad \leq C \frac{\varepsilon^{N}}{r^{N-2}} \int_{0}^{r} \frac{\rho^{N-1}}{\left(\varepsilon^{2}+\rho^{2}\right)^{\frac{N+2}{2}}} d \rho \\
& \quad \leq C \frac{\varepsilon^{N}}{r^{N-2}}\left(\int_{0}^{\varepsilon} \frac{\rho^{N-1}}{\varepsilon^{N+2}} d \rho+\int_{\varepsilon}^{r} \frac{\rho^{N-1}}{\rho^{N+2}} d \rho\right) \\
& \leq C\left(\frac{\varepsilon}{r}\right)^{N-2} .
\end{aligned}
$$

Therefore,

$$
\begin{aligned}
\int_{\Omega}\left|U_{\varepsilon}^{r}\right|^{2^{*}} d x & \geq \int_{\mathbb{R}^{N}}\left|U_{\varepsilon}\right|^{2^{*}} d x-C \varepsilon^{N} r^{-N}-C \varepsilon^{N-2} r^{-N+2} \\
& \geq S^{N / 2}-C\left(\frac{\varepsilon}{r}\right)^{N-2}
\end{aligned}
$$

that is, (2.5) holds. 
Now we assume that $\eta>0$. Note that $U_{\varepsilon}^{r}(x)<U_{\varepsilon}(x) \leq C \varepsilon^{-\frac{N-2}{2}}$, and $\left|\nabla U_{\varepsilon}^{r}(x)\right| \leq C \varepsilon^{-\frac{N}{2}}$, we have

$$
\begin{aligned}
\int_{\Omega}\left|\nabla\left(\xi_{\eta}(x-y) U_{\varepsilon}^{r}(x)\right)\right|^{2} d x \leq & \int_{\Omega}\left|\nabla U_{\varepsilon}^{r}\right|^{2} d x+\int_{\Omega}\left|\nabla \xi_{\eta}(x-y)\right|^{2}\left|U_{\varepsilon}^{r}(x)\right|^{2} d x \\
& +2 \int_{\Omega}\left|\xi_{\eta}(x-y) U_{\varepsilon}^{r}(x) \nabla \xi_{\eta}(x-y) \nabla U_{\varepsilon}^{r}(x)\right| d x \\
\leq & S^{N / 2}-C\left(\frac{\varepsilon}{r}\right)^{N-2}+C \int_{\frac{\eta}{2} \leq|x-y| \leq \eta} \frac{4}{\eta^{2}} \varepsilon^{-(N-2)} d x \\
& +C \int_{\frac{\eta}{2} \leq|x-y| \leq \eta} \frac{2}{\eta} \varepsilon^{-\frac{N-2}{2}} \varepsilon^{-\frac{N}{2}} d x \\
\leq & S^{N / 2}-C\left(\frac{\varepsilon}{r}\right)^{N-2}+C\left(\frac{\eta}{\varepsilon}\right)^{N-2} .
\end{aligned}
$$

Hence, (2.4) holds. Similarly

$$
\begin{aligned}
& \int_{\Omega}\left|\xi_{\eta}(x-y) U_{\varepsilon}^{r}(x)\right|^{2^{*}} d x \\
& \quad=\int_{\Omega}\left|U_{\varepsilon}^{r}\right|^{2^{*}} d x-\int_{\Omega}\left(1-\xi_{\eta}(x-y)^{2^{*}}\right)\left|U_{\varepsilon}^{r}\right|^{2^{*}} d x \\
& \quad \geq S^{N / 2}-C\left(\frac{\varepsilon}{r}\right)^{N-2}-C \int_{|x-y| \leq \eta} \varepsilon^{-N} d x \\
& \quad \geq S^{N / 2}-C\left(\frac{\varepsilon}{r}\right)^{N-2}-C\left(\frac{\eta}{\varepsilon}\right)^{N-2},
\end{aligned}
$$

that is, (2.5) holds.

Remark 2.1. Let $\eta=0$, the following similar estimate

$$
\int_{\Omega}\left|U_{\varepsilon}^{r}\right|^{2^{*}} d x \geq S^{N / 2}-C \varepsilon^{N} r^{-N}
$$

which is different from the inequality (2.5), is not true. Indeed, note that $\varepsilon^{N-2} r^{-N+2} \gg \varepsilon^{N} r^{-N}$ as $\varepsilon$ small enough. Assume that (2.6) holds, then together with (2.4) and let $\varepsilon$ small enough, we get that

$$
\frac{\int_{\Omega}\left|\nabla U_{\varepsilon}^{r}\right|^{2} d x}{\left(\int_{\Omega}\left|U_{\varepsilon}^{r}\right|^{2^{*}} d x\right)^{\frac{2}{2^{*}}}} \leq \frac{S^{N / 2}-C \varepsilon^{N-2} r^{-N+2}}{\left(S^{N / 2}-C \varepsilon^{N} r^{-N}\right)^{\frac{2}{2^{*}}}}<S,
$$

which is a contradiction.

Now, we let $\varepsilon_{r}=r^{\frac{N+2}{2}}$, and define $u_{r}=U_{\varepsilon_{r}}^{r}$, then it is easy to see that $u_{r}$ is continuous in $H_{0}^{1}(\Omega)$ with respect to $r \in\left(0, r_{2}\right]=\left(0, \frac{1}{6 m}\right]$. Let $\eta \in\left[0, r^{N+1}\right]$, the following lemma holds.

Lemma 2.8. There exists $C_{3}>0$ and $m_{1} \in \mathbb{N}$ such that for any $m \geq m_{1}$ and any $y \in \Omega$,

$$
\sup _{t \geq 0} I\left(t \xi_{\eta}(x-y) u_{r}(x)\right) \begin{cases}<\frac{1}{N} S^{N / 2} & \text { if } r \in\left(0, r_{2}\right], \\ \leq S_{m} & \text { if } r \in\left[\frac{r_{2}}{2}, r_{2}\right],\end{cases}
$$


where $S_{m}=\frac{1}{N} S^{N / 2}-C_{3} m^{-(N+2)}, S_{m}+C_{2} m^{-\frac{N(N-2)}{2}}<\frac{1}{N} S^{N / 2}$ and $C_{2}$ is defined in Lemma 2.6.

Proof. Let $r \leq r_{2}$. Note that $\eta \in\left[0, r^{N+1}\right]$, by Lemma 2.7 we have that

$$
\begin{gathered}
\int_{\Omega}\left|\nabla\left(\xi_{\eta}(x-y) u_{r}(x)\right)\right|^{2} d x \leq S^{N / 2}-C\left(\frac{\varepsilon_{r}}{r}\right)^{N-2}+C\left(\frac{\eta}{\varepsilon_{r}}\right)^{N-2} \\
\leq S^{N / 2}+C r^{\frac{N(N-2)}{2}}\left(\leq S^{N / 2}+C m^{-\frac{N(N-2)}{2}} \quad \text { if } r \in\left[\frac{r_{2}}{2}, r_{2}\right]\right),
\end{gathered}
$$

and

$$
\begin{aligned}
& \int_{\Omega}\left|\xi_{\eta}(x-y) u_{r}(x)\right|^{2^{*}} d x \geq S^{N / 2}-C\left(\frac{\varepsilon_{r}}{r}\right)^{N-2}-C\left(\frac{\eta}{\varepsilon_{r}}\right)^{N-2} \\
& \geq S^{N / 2}-C r^{\frac{N(N-2)}{2}}\left(\geq S^{N / 2}-C m^{-\frac{N(N-2)}{2}} \quad \text { if } r \in\left[\frac{r_{2}}{2}, r_{2}\right]\right),
\end{aligned}
$$

For $r$ small enough, we have $\varepsilon_{r}<\frac{r}{2}$ and $U_{\varepsilon_{r}}(x) \geq 2 A_{0}\left(\frac{\varepsilon_{r}}{\varepsilon_{r}^{2}+r^{2}}\right)^{\frac{N-2}{2}}$ for $|x| \leq \frac{r}{2}$. That is $u_{r}(x)=U_{\varepsilon_{r}}(x)-A_{0}\left(\frac{\varepsilon_{r}}{\varepsilon_{r}^{2}+r^{2}}\right)^{\frac{N-2}{2}} \geq \frac{1}{2} U_{\varepsilon_{r}}(x)$ for $|x| \leq \frac{r}{2}$. Hence, if $\eta=0$,

$$
\begin{aligned}
\int_{\Omega}\left|u_{r}(x)\right|^{2} d x & \geq \int_{B\left(0, \varepsilon_{r}\right)}\left|u_{r}(x)\right|^{2} d x \\
& \geq \frac{1}{4} \int_{B\left(0, \varepsilon_{r}\right)}\left|U_{\varepsilon_{r}}(x)\right|^{2} d x \\
& \geq C \int_{0}^{\varepsilon_{r}} \rho^{N-1}\left(\frac{\varepsilon_{r}}{2 \varepsilon_{r}^{2}}\right)^{N-2} d \rho \\
& \geq C \varepsilon_{r}^{2}=C r^{N+2}\left(\geq C m^{-(N+2)} \quad \text { if } r \in\left[\frac{r_{2}}{2}, r_{2}\right]\right) .
\end{aligned}
$$

Similarly, if $0<\eta \leq r^{N+1}$, we also have

$$
\begin{aligned}
& \int_{\Omega}\left|\xi_{\eta}(x-y) u_{r}(x)\right|^{2} d x \\
& \quad=\int_{\Omega}\left|u_{r}\right|^{2} d x-\int_{\Omega}\left(1-\xi_{\eta}(x-y)^{2}\right)\left|u_{r}\right|^{2} d x \\
& \quad \geq C r^{N+2}-\int_{|x-y| \leq \eta} \varepsilon_{r}^{-(N-2)} d x \\
& \quad \geq C r^{N+2}\left(\geq C m^{-(N+2)} \quad \text { if } r \in\left[\frac{r_{2}}{2}, r_{2}\right]\right) .
\end{aligned}
$$

For any constants $B_{1}, B_{2}>0$, we have that

$$
\max _{t \geq 0}\left(\frac{t^{2}}{2} B_{1}-\frac{t^{2^{*}}}{2^{*}} B_{2}\right)=\frac{1}{N} B_{1}\left(\frac{B_{1}}{B_{2}}\right)^{\frac{N-2}{2}}=\frac{1}{N}\left(\frac{B_{1}}{B_{2}^{2 / 2^{*}}}\right)^{\frac{N}{2}} .
$$


By (2.8)-(2.12) we have

$$
\begin{aligned}
I\left(t \xi_{\eta}(x-y) u_{r}(x)\right)= & \frac{t^{2}}{2} \int_{\Omega}\left(\left|\nabla\left(\xi_{\eta}(x-y) u_{r}(x)\right)\right|^{2}-\lambda\left(\xi_{\eta}(x-y) u_{r}(x)\right)^{2}\right) d x \\
& -\frac{t^{2^{*}}}{2^{*}} \int_{\Omega}\left|\xi_{\eta}(x-y) u_{r}(x)\right|^{2^{*}} d x \\
\leq & \frac{t^{2}}{2}\left(S^{N / 2}+C r^{\frac{N(N-2)}{2}}-\lambda C r^{N+2}\right) \\
& -\frac{t^{2^{*}}}{2^{*}}\left(S^{N / 2}-C r^{\frac{N(N-2)}{2}}\right) \\
\leq & \frac{1}{N}\left(S^{N / 2}-C r^{N+2}\right)\left(\frac{S^{N / 2}-C r^{N+2}}{\left.S^{N / 2}-C r^{\frac{N(N-2)}{2}}\right)^{\frac{N-2}{2}},}\right.
\end{aligned}
$$

since $N \geq 5$, we have $N+2<\frac{N(N-2)}{2}$. Note (2.3), there exists an $m_{1} \geq m_{0}$ such that for any $m \geq m_{1}$, we have $r \leq \frac{1}{6 m}$ and

$$
\begin{aligned}
& \sup _{t \geq 0} I\left(t \xi_{\eta}(x-y) u_{r}(x)\right) \leq \frac{1}{N} S^{N / 2}-C r^{N+2} \\
& \quad<\frac{1}{N} S^{N / 2}\left(\leq \frac{1}{N} S^{N / 2}-C_{3} m^{-(N+2)}, \quad \text { if } r \in\left[\frac{r_{2}}{2}, r_{2}\right]\right),
\end{aligned}
$$

and

$$
C_{2} m^{-\frac{N(N-2)}{2}}<C_{3} m^{-(N+2)}
$$

where $C_{3}$ is a constant independent of $m$. Let $S_{m}=\frac{1}{N} S^{N / 2}-C_{3} m^{-(N+2)}$, then Lemma 2.8 holds.

From now on, we fix $m \geq m_{1}$. Note $r_{1}=\frac{1}{2 m}, r_{2}=\frac{r_{1}}{3}=\frac{1}{6 m}$. Define $\eta_{r}=r^{N+1}$. Let

$$
\begin{aligned}
& M_{m}^{1}:=\left\{\xi_{\eta_{r}}(\cdot) u_{r}(\cdot+y): y \in \Omega, \operatorname{dist}(y, \partial \Omega)>r, r \in\left[\frac{1}{12 m}, \frac{1}{6 m}\right]\right\}, \\
& M_{m}^{2}:=\left\{u_{r}(\cdot+y): y \in \Omega, \operatorname{dist}(y, \partial \Omega)>r, r \in\left(0, \frac{1}{6 m}\right]\right\} .
\end{aligned}
$$

Then for any $u(x)=\xi_{\eta_{r}}(x) u_{r}(x+y) \in M_{m}^{1}$, we have $u \in H_{0}^{1}(\Omega), \operatorname{supp}(u) \subset$ $\overline{B(-y, r) \backslash B\left(0, \eta_{r} / 2\right)}$, and $\sup _{t \geq 0} I(t u(x))=\sup _{t \geq 0} I(t u(x-y)) \leq S_{m}$. Similarly, for any $u=u_{r}(\cdot+y) \in M_{m}^{2}$, we have $\operatorname{supp}(u) \subset \overline{B(-y, r)}$ and $\sup _{t \geq 0} I(t u(x))<$ $\frac{1}{N} S^{N / 2}$. We write $\mathbb{S}^{k}=\left\{x \in \mathbb{R}^{k+1}:|x|=1\right\}, \quad \mathbb{B}^{k}=\left\{x \in \mathbb{R}^{k}:|x| \leq 1\right\}$ for $k \in \mathbb{N}$. We consider $u^{ \pm}:=\max \{ \pm u, 0\}$. We have the following lemma.

Lemma 2.9. There exists an odd continuous map $h: \mathbb{S}^{N} \rightarrow H_{0}^{1}\left(B\left(0, r_{1}\right)\right)$ such that one of $h(\theta)^{ \pm}$belongs to $M_{m}^{1}$ and the other belongs to $M_{m}^{1} \cup M_{m}^{2}$ for every $\theta \in \mathbb{S}^{N}$, that is

$$
\sup _{t \geq 0} I(t h(\theta))<S_{m}+\frac{1}{N} S^{N / 2}, \quad \text { for any } \theta \in \mathbb{S}^{N} .
$$


Proof. Note $\eta_{r}=r^{N+1}$. For $y \in \mathbb{B}^{N}$, we set $t=|y|, \theta=\frac{y}{|y|}$ and define $\widetilde{h}: \mathbb{B}^{N} \rightarrow H_{0}^{1}\left(B\left(0, r_{1}\right)\right)$ by

$\widetilde{h}(y)= \begin{cases}u \frac{\eta_{r_{2}}}{2}(\cdot)-\xi_{\eta_{r_{2}}}(\cdot) u_{r_{2}}\left(\cdot+4 t r_{2} \theta\right) & \text { if } 0 \leq t \leq \frac{1}{2}, \\ u_{t\left(2 r_{2}-\eta_{r_{2}}\right)-r_{2}+\eta_{r_{2}}}\left(\cdot-2 r_{2}(2 t \theta-\theta)\right)-u_{r_{2}}\left(\cdot+2 r_{2} \theta\right) & \text { if } \frac{1}{2} \leq t \leq 1 .\end{cases}$

Since $\xi_{\eta_{r_{2}}}(x) \equiv 1$ for $|x| \geq \eta_{r_{2}}$, we see that $\xi_{\eta_{r_{2}}}(\cdot) u_{r_{2}}\left(\cdot+2 r_{2} \theta\right) \equiv u_{r_{2}}\left(\cdot+2 r_{2} \theta\right)$, and so $\widetilde{h}$ is continuous on $\mathbb{B}^{N}$. Moreover, $\widetilde{h}(y)^{+} \in M_{m}^{2}, \widetilde{h}(y)^{-} \in M_{m}^{1}$, and $\operatorname{supp}\left(\widetilde{h}(y)^{+}\right) \cap \operatorname{supp}\left(\widetilde{h}(y)^{-}\right)=\emptyset$. Notice that $\widetilde{h}$ is odd on $\mathbb{S}^{N-1}$, it induces an odd continuous map $h: \mathbb{S}^{N} \rightarrow H_{0}^{1}\left(B\left(0, r_{1}\right)\right)$ by

$$
h\left(x_{1}, \ldots, x_{N+1}\right)= \begin{cases}\widetilde{h}\left(x_{1}, \ldots, x_{N}\right) & \text { if } x_{N+1} \geq 0, \\ -\widetilde{h}\left(-x_{1}, \ldots,-x_{N}\right) & \text { if } x_{N+1} \leq 0,\end{cases}
$$

then one of $h(\theta)^{ \pm}$belongs to $M_{m}^{1}$ and the other belongs to $M_{m}^{1} \cup M_{m}^{2}$ for every $\theta \in \mathbb{S}^{N}$, and $\operatorname{supp}\left(h(\theta)^{+}\right) \cap \operatorname{supp}\left(h(\theta)^{-}\right)=\emptyset$, that is

$$
\sup _{t \geq 0} I(t h(\theta)) \leq \sup _{t \geq 0} I\left(t h(\theta)^{+}\right)+\sup _{t \geq 0} I\left(t h(\theta)^{-}\right)<S_{m}+\frac{1}{N} S^{N / 2},
$$

hence (2.13) holds.

Lemma 2.10. There exists an odd continuous map $\bar{h}: \mathbb{R}^{n+N+2} \rightarrow H_{0}^{1}(\Omega)$ such that $\lim _{|x| \rightarrow+\infty} I(\bar{h}(x))=-\infty \quad$ and $\quad \tilde{S}:=\sup _{u \in \bar{h}\left(\mathbb{R}^{n+N+2}\right)} I(u)<\frac{2}{N} S^{N / 2}$.

Proof. Note that $e_{i}^{m} \in H_{0}^{1}\left(\Omega \backslash B\left(0, \frac{1}{m}\right)\right)$, we define an odd continuous map $h_{1}: \mathbb{R}^{n} \rightarrow H_{0}^{1}\left(\Omega \backslash B\left(0, \frac{1}{m}\right)\right)$ by $h_{1}\left(x_{1}, \ldots, x_{n}\right):=\sum_{i=1}^{n} x_{i} e_{i}^{m}$. Then by (2.3) it follows that $\sup _{u \in h_{1}\left(\mathbb{R}^{n}\right)} I(u) \leq C_{2} m^{-\frac{N(N-2)}{2}}$. Since all norms are equivariant in $V_{m}^{-}$, it is easy to see that $\lim _{|x| \rightarrow+\infty} I\left(h_{1}(x)\right)=-\infty$. By Lemma 2.9, there exists an odd continuous map

$$
h: \mathbb{S}^{N} \rightarrow H_{0}^{1}\left(B\left(0, r_{1}\right)\right)=H_{0}^{1}\left(B\left(0, \frac{1}{2 m}\right)\right)
$$

such that one of $h(\theta)^{ \pm}$belongs to $M_{m}^{1}$ and the other belongs to $M_{m}^{1} \cup M_{m}^{2}$ for every $\theta \in \mathbb{S}^{N}$. Fix $y_{0} \in \Omega$ with $\left|y_{0}\right|=\frac{3}{4 m}$, and let $v_{0}(x)=\xi_{\eta_{r_{2}}}(x) u_{r_{2}}\left(x+y_{0}\right)$ $\in M_{m}^{1}$. Then $v_{0} \in H_{0}^{1}\left(B\left(0, \frac{1}{m}\right) \backslash B\left(0, \frac{1}{2 m}\right)\right) \cap M_{m}^{1}$, and $\sup _{t \geq 0} I\left(t v_{0}\right) \leq S_{m}$. Let

$$
Z:=\left(\mathbb{S}^{N} \times[-1,1]\right) \cup\left(\mathbb{B}^{N+1} \times\{-1,1\}\right) \subset \mathbb{R}^{N+1} \times \mathbb{R} \equiv \mathbb{R}^{N+2}
$$

and extend $h$ to a map $h_{2}: Z \rightarrow H_{0}^{1}\left(B\left(0, \frac{1}{m}\right)\right)$ as follows: for $\theta \in \mathbb{S}^{N}, s \in[0,1]$ and $t \in[-1,1]$ we define

$$
h_{2}(s \theta, t):= \begin{cases}(1-t) h(\theta)^{-}+(1+t) h(\theta)^{+} & \text {if } s=1, \\ 2 \operatorname{sh}(\theta)^{+}+(1-s) v_{0} & \text { if } t=1, \\ 2 \operatorname{sh}(\theta)^{-}-(1-s) v_{0} & \text { if } t=-1 .\end{cases}
$$

Then we extend $h_{2}$ radially to a map $h_{3}: \mathbb{R}^{N+2} \rightarrow H_{0}^{1}\left(B\left(0, \frac{1}{m}\right)\right)$ by

$$
h_{3}(t z):=t h_{2}(z) \quad \text { for } \quad z \in Z, \quad t \geq 0 .
$$


By construction, $h_{3}$ is an odd continuous map. Moreover, one of $h_{3}(z)^{ \pm}$ belongs to $M_{m}^{1}$ and the other belongs to $M_{m}^{1} \cup M_{m}^{2}$ for any $z \in \mathbb{R}^{N+2}$, and $\operatorname{supp}\left(h_{3}(z)^{+}\right) \cap \operatorname{supp}\left(h_{3}(z)^{-}\right)=\emptyset$, hence we have

$$
\sup _{u \in h_{3}\left(\mathbb{R}^{N+2}\right)} I(u)<S_{m}+\frac{1}{N} S^{N / 2}, \quad \lim _{|x| \rightarrow+\infty} I\left(h_{3}(x)\right)=-\infty .
$$

Now we define $\bar{h}: \mathbb{R}^{n+N+2} \rightarrow H_{0}^{1}(\Omega)$ by $\bar{h}(y, z)=h_{1}(y)+h_{3}(z)$ for all $y \in \mathbb{R}^{n}$, $z \in \mathbb{R}^{N+2}$. Then $\bar{h}$ is an odd continuous map, satisfies $\lim _{|x| \rightarrow+\infty} I(\bar{h}(x))=-\infty$ and

$$
\begin{aligned}
\sup _{(y, z) \in \mathbb{R}^{n+N+2}} I(\bar{h}(y+z)) & \leq \sup _{y \in \mathbb{R}^{n}} I\left(h_{1}(y)\right)+\sup _{z \in \mathbb{R}^{N+2}} I\left(h_{3}(z)\right) \\
& <C_{2} m^{-\frac{N(N-2)}{2}}+S_{m}+\frac{1}{N} S^{N / 2}<\frac{2}{N} S^{N / 2} .
\end{aligned}
$$

That is, $\tilde{S}<\frac{2}{N} S^{N / 2}$.

Lemma 2.11. $\beta_{n+N+2}<2^{2 / N} S$.

Proof. Let $A:=\left\{u \in \bar{h}\left(\mathbb{R}^{n+N+2}\right):\|u\|_{2^{*}}=1\right\}$. Then $A \subset M, \gamma(A) \geq n+$ $N+2$. In fact, assume that $\gamma(A)=k<n+N+2$, then there exists an odd continuous map $g: A \rightarrow \mathbb{R}^{k} \backslash\{0\}$. Let $O:=\left\{x \in \mathbb{R}^{n+N+2}:\|\bar{h}(x)\|_{2^{*}}<1\right\}$, then $O$ is a symmetric open bounded subset of $\mathbb{R}^{n+N+2}, 0 \in O$ and $\bar{h}(\partial O)=A$, that is, $g \circ \bar{h}: \partial O \subset \mathbb{R}^{n+N+2} \backslash\{0\} \rightarrow \mathbb{R}^{k} \backslash\{0\}$ is an odd continuous map, which contradicts with the Borsuk-Ulam Theorem. So $\gamma(A) \geq n+N+2$, that is $A \in \Sigma_{n+N+2}$. For any $u \in A$, by (2.12) and Lemma 2.10 we get

$$
\begin{aligned}
\frac{2}{N} S^{N / 2}>\tilde{S} & \geq \max _{t>0} I(t u)=\max _{t>0}\left(\frac{t^{2}}{2} \int_{\Omega}\left(|\nabla u|^{2}-\lambda u^{2}\right) d x-\frac{t^{2^{*}}}{2^{*}} \int_{\Omega}|u|^{2^{*}} d x\right) \\
& =\frac{1}{N}\left(\frac{\int_{\Omega}\left(|\nabla u|^{2}-\lambda u^{2}\right) d x}{\left(\int_{\Omega}|u|^{2 *} d x\right)^{2 / 2^{*}}}\right)^{N / 2}=\frac{1}{N} J(u)^{N / 2},
\end{aligned}
$$

That is, $\sup _{u \in A} J(u) \leq(N \tilde{S})^{2 / N}<2^{2 / N} S$. By the definition of $\beta_{n+N+2}$, we see that $\beta_{n+N+2}<2^{2 / N} S$.

Proof of Theorem 1.1 If $K^{\beta}$ is infinite for some $0<\beta<2^{2 / N} S, J$ has infinitely many critical points, and so do $I$. Hence, we assume that $K^{\beta}$ is finite for all $0<\beta<2^{2 / N} S$. By Lemma 2.11, we have

$$
0<\beta_{n+1}<\beta_{n+2}<\cdots<\beta_{n+N+2}<2^{2 / N} S .
$$

Let $k_{0} \in \mathbb{N}$ be such that $\beta_{n+k_{0}}<S \leq \beta_{n+k_{0}+1}$. By Lemma 2.3, $J$ has at least

$$
\max \left\{k_{0}, N+2-\left(k_{0}+1\right)\right\} \geq\left\lceil\frac{N+1}{2}\right\rceil
$$

nontrivial critical points. Hence, $I$ has at least $\left\lceil\frac{N+1}{2}\right\rceil$ nontrivial critical points. This completes the proof. 


\section{Proof of Theorem 1.2}

In this section, we assume that $N \geq 3$ and $\lambda_{n} \leq \lambda<\lambda_{n+1}$. The following lemma is crucial.

Lemma 3.1. If $u$ is a nontrivial solution of (1.1), then

$$
\begin{aligned}
\|u\|_{2^{*}} & >\left(1-\frac{\lambda}{\lambda_{n+1}}\right)^{\frac{N-2}{4}} S^{\frac{N-2}{4}}, \\
\|u\| & >\left(1-\frac{\lambda}{\lambda_{n+1}}\right)^{\frac{N-2}{4}} S^{\frac{N}{4}}, \\
I(u) & >\frac{1}{N}\left(1-\frac{\lambda}{\lambda_{n+1}}\right)^{N / 2} S^{N / 2} .
\end{aligned}
$$

Proof. Assume that $u$ is a nontrivial solution of (1.1). Since $H_{0}^{1}(\Omega)=V^{-} \oplus V^{+}$, we have $u=w+v$, where $w \in V^{-}, v \in V^{+}$. Since $I^{\prime}(w) w<0$ if $w \neq 0$, then we have that $v \neq 0$. By $I^{\prime}(u) w=0$ it follows that $\int_{\Omega}|u|^{2^{*}-2} u w d x=$ $\|w\|^{2}-\lambda\|w\|_{2}^{2} \leq 0$, that is, $\int_{\Omega}|u|^{2^{*}-2} v w d x+\int_{\Omega}|u|^{2^{*}-2} w^{2} d x \leq 0$. Therefore, $\int_{\Omega}|u|^{2^{*}-2} v w d x \leq 0$. On the other hand, from $I^{\prime}(u) v=0$ it follows that

$$
\int_{\Omega}|u|^{2^{*}-2} u v d x=\|v\|^{2}-\lambda\|v\|_{2}^{2} \geq\left(1-\frac{\lambda}{\lambda_{n+1}}\right)\|v\|^{2} .
$$

Hence,

$$
\begin{aligned}
\left(1-\frac{\lambda}{\lambda_{n+1}}\right)\|v\|^{2} & \leq \int_{\Omega}|u|^{2^{*}-2} v w d x+\int_{\Omega}|u|^{2^{*}-2} v^{2} d x \\
& \leq \int_{\Omega}|u|^{2^{*}-2} v^{2} d x \leq\|u\|_{2^{*}}^{2^{*}-2}\|v\|_{2^{*}}^{2} \\
& <S^{-1}\|u\|_{2^{*}}^{2^{*}-2}\|v\|^{2} .
\end{aligned}
$$

Since $v \neq 0$, we get that

$$
\|u\|_{2^{*}}>\left(1-\frac{\lambda}{\lambda_{n+1}}\right)^{\frac{1}{2^{*}-2}} S^{\frac{1}{2^{*}-2}}=\left(1-\frac{\lambda}{\lambda_{n+1}}\right)^{\frac{N-2}{4}} S^{\frac{N-2}{4}} .
$$

Hence,

$$
\begin{aligned}
& \|u\|>S^{\frac{1}{2}}\|u\|_{2^{*}}>\left(1-\frac{\lambda}{\lambda_{n+1}}\right)^{\frac{N-2}{4}} S^{\frac{N}{4}} \\
& I(u)=I(u)-\frac{1}{2} I^{\prime}(u) u=\frac{1}{N}\|u\|_{2^{*}}^{2^{*}}>\frac{1}{N}\left(1-\frac{\lambda}{\lambda_{n+1}}\right)^{N / 2} S^{N / 2} .
\end{aligned}
$$

Let $K:=\left\{u \in H_{0}^{1}(\Omega): u \neq 0, I^{\prime}(u)=0\right\}$. Define $c:=\inf _{u \in K} I(u)$. We have the following theorem.

Theorem 3.1. If problem (1.1) has a nontrivial solution $u$ with $I(u)<\frac{1}{N} S^{N / 2}$, then there exists $u_{0} \in K$, such that $I\left(u_{0}\right)=c$, i.e., $u_{0}$ is a ground state solution of problem (1.1). 
Proof. Since problem (1.1) has a nontrivial solution $u$ with $I(u)<\frac{1}{N} S^{N / 2}$, we get that $K \neq \emptyset$ and $c<\frac{1}{N} S^{N / 2}$. Let $u_{k} \in K$ such that $\lim _{k \rightarrow \infty} I\left(u_{k}\right)=c$. Then $\left(u_{k}\right)$ is a $(P S)_{c}$ sequence for $I$. Since $I$ satisfies $(P S)_{a}$ condition for $a<\frac{1}{N} S^{N / 2}[24,25]$, it is well know that $\left(u_{k}\right)$ has a convergent subsequence, still denoted by $\left(u_{k}\right)$. So we may assume $u_{k} \rightarrow u_{0}$ in $H$ and $I^{\prime}\left(u_{0}\right)=0$. By Lemma 3.1, $\left\|u_{0}\right\|=\lim _{k \rightarrow \infty}\left\|u_{k}\right\| \geq\left(1-\frac{\lambda}{\lambda_{n+1}}\right)^{\frac{N-2}{4}} S^{\frac{N}{4}}$, that is, $u_{0} \neq 0$. Therefore, $u_{0} \in K, I\left(u_{0}\right)=c$, i.e., $u_{0} \in K$ is a ground state solution to problem (1.1).

Proof of Theorem 1.2. When $N=4, \lambda \neq \lambda_{n}$ or $N \geq 5$, problem (1.1) has a nontrivial solution $u$ with $I(u)<\frac{1}{N} S^{N / 2}[7,31]$. When $N=3, \lambda_{n+1}-S|\Omega|^{-2 / N}<$ $\lambda<\lambda_{n+1}$, problem (1.1) has a nontrivial solution $u$ with $I(u)<\frac{1}{N} S^{N / 2}$ [8]. Therefore, by Lemma 3.1 and Theorem 3.1, problem (1.1) has a ground state solution $u$ with $I(u)>\frac{1}{N}\left(1-\frac{\lambda}{\lambda_{n+1}}\right)^{N / 2} S^{N / 2}$.

\section{Proof of Theorems 1.3 and 1.4}

First we give a sketch of a proof of Theorem 1.3. Let $N \geq 10$, and $\widetilde{\lambda}_{n} \leq \lambda<$ $\widetilde{\lambda}_{n+1}$ for some $n \geq 1$. Denote $2^{\sharp}=2 N /(N-4)$. We set $\widetilde{H}=H_{0}^{2}(\Omega)$ (resp. $\widetilde{H}=H^{2}(\Omega) \cap H_{0}^{1}(\Omega)$ ) for problem (1.2) (resp. (1.3)). In each case, we define $(u, v)=\int_{\Omega} \Delta u \Delta v d x$ and $\|u\|^{2}=(u, u)$ for each $u, v \in \widetilde{H}$. Then we know that $\widetilde{H}$ is a Hilbert space and its norm is equivalent to the standard $H^{2}(\Omega)$-norm. Let $\lambda>0$, and let $\tilde{I}$ be a functional defined by

$$
\tilde{I}(u)=\frac{1}{2} \int_{\Omega}\left(|\Delta u|^{2}-\lambda|u|^{2}\right) d x-\frac{1}{2^{\sharp}} \int_{\Omega}|u|^{2^{\sharp}} d x \quad \text { for } u \in \widetilde{H} .
$$

We know that each critical point of $\tilde{I}$ is a solution of (1.2) in the case $\widetilde{H}=H_{0}^{2}(\Omega)$ (resp. a solution of $(1.3)$ in the case $\widetilde{H}=H^{2}(\Omega) \cap H_{0}^{1}(\Omega)$ ). As in Sect. 2, we consider a new functional

$$
\tilde{J}(u):=\frac{\int_{\Omega}\left(|\Delta u|^{2}-\lambda u^{2}\right) d x}{\left(\int_{\Omega}|u|^{2^{\sharp}} d x\right)^{2 / 2^{\sharp}}}=\int_{\Omega}\left(|\Delta u|^{2}-\lambda u^{2}\right) d x
$$

defined on $\widetilde{M}:=\left\{u \in \widetilde{H}:\|u\|_{2^{\sharp}}=1\right\}$. Then $\widetilde{M} \subset \widetilde{H}$ is a complete Hilbert manifold, invariant under the involution $u \rightarrow-u$. Moreover, we have that $\tilde{J} \in C^{1}(\widetilde{M}, \mathbb{R})$, and if $u \in \widetilde{M}$ is a critical point of $\tilde{J}$ with $\tilde{J}(u)=\beta>0$, if and only if $\tilde{u}:=\beta^{\frac{1}{2^{\sharp}-2}} u$ is a critical point of $\tilde{I}$ with $\tilde{I}(\tilde{u})=\frac{2}{N} \beta^{N / 4}$. $\left(u_{m}\right)$ is a $(P S)_{\beta}$ sequence for $\tilde{J}$ if and only if the sequence $\left(\tilde{u}_{m}\right)$, where $\tilde{u}_{m}:=\beta^{\frac{1}{2^{\sharp}-2}} u_{m}$, is a $(P S)_{\tilde{\beta}}$ sequence for $\tilde{I}$ with $\tilde{\beta}=\frac{2}{N} \beta^{N / 4}$. Denote $\widetilde{K}^{\beta}:=\left\{u \in \widetilde{M}: \tilde{J}^{\prime}(u)=\right.$ $0, \tilde{J}(u)=\beta\}$. Now, for $j \in \mathbb{N}, j \geq n+1$, we define $\Sigma_{j}:=\{A \subset \widetilde{M}: A=$ $-A=\bar{A}, \gamma(A) \geq j$, where $\gamma$ denotes the usual Krasnoselskii genus, and consider

$$
\beta_{j}:=\inf _{A \in \Sigma_{j}} \sup _{u \in A} \tilde{J}(u)
$$


As in Sect. 2, one can prove that for all $j \geq n+1$ the energy level $\beta_{j}$ is positive, and that there exists a $(P S)_{\beta_{j}}$ sequence $\left(u_{m}\right)$ for $\tilde{J}$ (see Lemma 2.2). For each $\varepsilon>0$ and $y \in \mathbb{R}^{N}$, we set

$$
\widetilde{U}_{\varepsilon, y}(x)=C_{N}\left(\frac{\varepsilon}{\varepsilon^{2}+|x-y|^{2}}\right)^{(N-4) / 2} \text { for } x \in \mathbb{R}^{N},
$$

where $C_{N}=((N-4)(N-2) N(N+2))^{(N-4) / 8}$. We denote by $\widetilde{S}$ the best constant for the Sobolev embedding from $\mathcal{D}^{2,2}\left(\mathbb{R}^{N}\right)$ into $L^{\frac{2 N}{N-4}}\left(\mathbb{R}^{N}\right)$, where $\mathcal{D}^{2,2}\left(\mathbb{R}^{N}\right)$ is the completion of $C_{0}^{\infty}\left(\mathbb{R}^{N}\right)$ with respect to the norm $\left(\int_{\mathbb{R}^{N}}|\Delta u|^{2} d x\right)^{1 / 2}$. Then $\widetilde{U}_{\varepsilon, y}$ satisfies the equation $\Delta^{2} u=|u|^{2^{\sharp}-2} u$ on $\mathbb{R}^{N}$ and

$$
\int_{\mathbb{R}^{N}}\left|\Delta \widetilde{U}_{\varepsilon, y}\right|^{2} d x=\int_{\mathbb{R}^{N}}\left|\widetilde{U}_{\varepsilon, y}\right|^{2^{\sharp}} d x=\widetilde{S}^{N / 4} .
$$

Let

$$
\widetilde{E}:=\left\{\widetilde{U}_{\varepsilon, y}: \varepsilon>0, y \in \mathbb{R}^{N}\right\} .
$$

From [17] we see that $\widetilde{E}$ contains all positive solutions of the equation $\Delta^{2} u=$ $|u|^{2^{\sharp}-2} u$ on $\mathbb{R}^{N}$. Using similar proofs of Lemma 2.3 in Sect. 2, the following lemma holds directly from Lemma 13 of [11].

Lemma 4.1. Let $\left(u_{m}\right)$ be a $(P S)_{\beta_{j}}$ sequence for $\tilde{J}$. Up to a subsequence, the following properties hold.

(a) If $0<\beta_{j}<\widetilde{S}$, then $\left(u_{m}\right)$ converges in $\widetilde{M}$ and $\beta_{j}$ is a critical value of $\tilde{J}$.

(b) If $\widetilde{S}<\beta_{j}<2^{4 / N} \widetilde{S}$, then one of the following conclusions holds:

(b.1) $\quad\left(u_{m}\right)$ converges in $\widetilde{M}$, that is $\beta_{j}$ is a critical value of $\tilde{J}$.

(b.2) There is a critical point $u$ of $\tilde{J}$ with $\tilde{J}(u)=\beta_{\sharp}=\left(\beta_{j}^{N / 4}-\widetilde{S}^{N / 4}\right)^{4 / N} \in$ $(0, \widetilde{S})$ such that

$\operatorname{dist}\left(\beta_{j}^{\frac{1}{2^{\sharp}-2}} u_{m}-\beta_{\sharp}^{\frac{1}{2^{\sharp}-2}} u, \widetilde{E}\right) \rightarrow 0$ or $\operatorname{dist}\left(\beta_{j}^{\frac{1}{2^{\sharp}-2}} u_{m}-\beta_{\sharp}^{\frac{1}{2^{\sharp}-2}} u,-\widetilde{E}\right) \rightarrow 0$.

(c) If $\beta_{j}=\widetilde{S}$, then one of the following conclusions holds:

(c.1) $\left(u_{m}\right)$ converges in $\widetilde{M}$ and $\beta_{j}$ is a critical value of $\tilde{J}$.

(c.2) $\operatorname{dist}\left(\beta_{j}^{\frac{1}{2^{\sharp}-2}} u_{m}, \widetilde{E}\right) \rightarrow 0$ or $\operatorname{dist}\left(\beta_{j}^{\frac{1}{2^{\sharp}-2}} u_{m},-\widetilde{E}\right) \rightarrow 0$.

From Lemma 4.1 and similar proofs of Lemma 2.4, we can show that if $0<\beta_{j}=\beta_{j+1}<2^{4 / N} \widetilde{S}$, then $\widetilde{K}^{\beta_{j}}$ is infinite. We choose a complete orthonormal basis $\left\{\psi_{i}\right\}_{i=1}^{\infty}$ of $\widetilde{H}$ such that each $\psi_{i} \in \widetilde{H}$ satisfies $\Delta^{2} \psi_{i}=\widetilde{\lambda}_{i} \psi_{i}$ in $\Omega$. Then we may choose $C_{4}>0$ such that $\left|\psi_{i}(x)\right| \leq C_{4},\left|\nabla \psi_{i}(x)\right| \leq C_{4}$, $\left|\Delta \psi_{i}(x)\right| \leq C_{4}$ for each $i=1, \ldots, n$. Without loss of generality, we may assume that $0 \in \Omega$. Then we have $B\left(0, \frac{2}{m}\right) \in \Omega$ for $m$ large enough. Let $\psi_{i}^{m}:=\zeta_{m} \psi_{i}$ and $\widetilde{V}_{m}^{-}:=\operatorname{span}\left\{\psi_{1}^{m}, \ldots, \psi_{n}^{m}\right\}$, where $\zeta_{m} \in C_{0}^{\infty}\left(\mathbb{R}^{N}\right)$ such that $0 \leq \zeta_{m} \leq 1$, $\left|\nabla \zeta_{m}\right| \leq 2 m,\left|\Delta \zeta_{m}\right| \leq C m^{2}, \zeta_{m}(x)=0$ if $|x| \leq \frac{1}{m}$ and $\zeta_{m}(x)=1$ if $|x| \geq \frac{2}{m}$. Then we have

$$
\left\|\psi_{i}^{m}-\psi_{i}\right\|^{2}=\int_{\Omega}\left|\Delta\left(\zeta_{m} \psi_{i}\right)-\Delta \psi_{i}\right|^{2} d x
$$




$$
\begin{aligned}
= & \int_{\Omega}\left|\Delta \zeta_{m} \psi_{i}+2 \nabla \zeta_{m} \nabla \psi_{i}+\left(\zeta_{m}-1\right) \Delta \psi_{i}\right|^{2} d x \\
\leq & \int_{B\left(0, \frac{2}{m}\right)}\left(\left|\Delta \zeta_{m}\right|^{2}\left|\psi_{i}\right|^{2}+4\left|\Delta \zeta_{m} \psi_{i} \nabla \zeta_{m} \nabla \psi_{i}\right|\right. \\
& +2\left|\Delta \zeta_{m} \psi_{i} \Delta \psi_{i}\right|+4\left|\nabla \zeta_{m}\right|^{2}\left|\nabla \psi_{i}\right|^{2} \\
& \left.+4\left|\nabla \zeta_{m} \nabla \psi_{i} \Delta \psi_{i}\right|+\left|\zeta_{m}-1\right|^{2}\left|\Delta \psi_{i}\right|^{2}\right) d x \\
\leq & C \int_{B\left(0, \frac{2}{m}\right)}\left(m^{4}+m^{3}+m^{2}+m^{2}+m+1\right) d x \\
\leq & C m^{-N+4} .
\end{aligned}
$$

Then by similar proofs of Lemma 2.5 and Lemma 2.6, we see that

$$
\begin{aligned}
\max _{\left\{u \in \widetilde{V}_{m}^{-}:\|u\|_{2}=1\right\}}\|u\|^{2} \leq \widetilde{\lambda}_{n}+C m^{-N+4}, \\
\sup _{u \in \widetilde{V}_{m}^{-}} I(u) \leq C_{5} m^{-\frac{N(N-4)}{4}} .
\end{aligned}
$$

We choose $\rho \in C_{0}^{\infty}\left(\mathbb{R}^{N}\right)$ satisfying $0 \leq \rho \leq 1,|\nabla \rho| \leq 2,|\Delta \rho| \leq C_{6}$ and

$$
\rho(x)= \begin{cases}1 & \text { if }|x| \leq 1 \\ 0 & \text { if }|x| \geq 2\end{cases}
$$

where $C_{6}$ is a constant. We denote $\widetilde{U}_{\varepsilon}:=\widetilde{U}_{\varepsilon, 0}=C_{N}\left(\frac{\varepsilon}{\varepsilon^{2}+|x|^{2}}\right)^{\frac{N-4}{2}}$ and let $r_{1}=\frac{1}{2 m}, r_{2}=\frac{r_{1}}{3}$. Then for any $r \in\left(0, r_{2}\right]=\left(0, \frac{1}{6 m}\right]$, we define $\rho_{r}(x)=\rho\left(\frac{2 x}{r}\right)$. Then $\rho_{r} \in C_{0}^{\infty}(\overline{B(0, r)}), 0 \leq \rho_{r} \leq 1,\left|\nabla \rho_{r}\right| \leq 4 r^{-1},\left|\Delta \rho_{r}\right| \leq 4 C_{6} r^{-2}$. Define $\widetilde{U}_{\varepsilon}^{r}=\rho_{r} \widetilde{U}_{\varepsilon}$, which is quite different from the definition of $U_{\varepsilon}^{r}$ in Sect. 2. Then $\widetilde{U}_{\varepsilon}^{r} \in \widetilde{H}$ with $\operatorname{supp}\left(\widetilde{U}_{\varepsilon}^{r}\right) \subset \overline{B(0, r)}$. Define $\widetilde{\xi}_{\eta}(x)=1-\rho_{\eta}(x)$ for $\eta>0$ and $\widetilde{\xi}_{\eta}(x) \equiv 1$ for $\eta=0$. Denote $\sigma_{\eta, y}(x)=\widetilde{\xi}_{\eta}(x-y)$ for convenience. Let $0 \leq 2 \eta<$ $\varepsilon<r / 2$, then the following lemma holds.

Lemma 4.2. There exists $C>0$, depending only on $N$, such that for any $y \in \Omega$, we have

$$
\begin{aligned}
& \int_{\Omega}\left|\Delta\left(\sigma_{\eta, y} \widetilde{U}_{\varepsilon}^{r}\right)\right|^{2} d x \leq \widetilde{S}^{N / 4}+C\left((\varepsilon / r)^{N-4}+(\eta / \varepsilon)^{N-4}\right), \\
& \int_{\Omega}\left|\sigma_{\eta, y} \widetilde{U}_{\varepsilon}^{r}\right|^{\sharp} d x \geq \widetilde{S}^{N / 4}-C\left((\varepsilon / r)^{N}+(\eta / \varepsilon)^{N}\right), \\
& \int_{\Omega}\left|\sigma_{\eta, y} \widetilde{U}_{\varepsilon}^{r}\right|^{2} d x \geq C \varepsilon^{4} .
\end{aligned}
$$

Proof. We may assume that $\eta>0$, since the case of $\eta=0$ is much simpler. First we show (4.3). We note that

$$
\begin{aligned}
& \left.\left|\int_{\Omega}\right| \Delta\left(\sigma_{\eta, y} \widetilde{U}_{\varepsilon}^{r}\right)\right|^{2} d x-\widetilde{S}^{N / 4} \mid \\
& \quad=\left.\left|\int_{\mathbb{R}^{N}}\right| \Delta\left(\sigma_{\eta, y} \rho_{r} \widetilde{U}_{\varepsilon}\right)\right|^{2} d x-\int_{\mathbb{R}^{N}}\left|\Delta \widetilde{U}_{\varepsilon}\right|^{2} d x \mid
\end{aligned}
$$




$$
\begin{aligned}
\leq & \int_{\mathbb{R}^{N}}\left|\sigma_{\eta, y}^{2} \rho_{r}^{2}-1\right|\left|\Delta \widetilde{U}_{\varepsilon}\right|^{2} d x \\
& +C\left(\int_{\mathbb{R}^{N}}\left|\Delta \sigma_{\eta, y}\right|^{2} \widetilde{U}_{\varepsilon}^{2} d x\right. \\
& +\int_{\mathbb{R}^{N}}\left|\Delta \rho_{r}\right|^{2} \widetilde{U}_{\varepsilon}^{2} d x+\int_{\mathbb{R}^{N}}\left|\nabla \sigma_{\eta, y}\right|^{2}\left|\nabla \widetilde{U}_{\varepsilon}\right|^{2} d x \\
& +\int_{\mathbb{R}^{N}}\left|\nabla \rho_{r}\right|^{2}\left|\nabla \widetilde{U}_{\varepsilon}\right|^{2} d x+\int_{\mathbb{R}^{N}} \widetilde{U}_{\varepsilon}\left|\Delta \sigma_{\eta, y}\right|\left|\Delta \widetilde{U}_{\varepsilon}\right| d x \\
& +\int_{\mathbb{R}^{N}} \widetilde{U}_{\varepsilon}\left|\Delta \rho_{r}\right|\left|\Delta \widetilde{U}_{\varepsilon}\right| d x+\int_{\mathbb{R}^{N}} \widetilde{U}_{\varepsilon}\left|\nabla \sigma_{\eta, y}\right|\left|\nabla \rho_{r}\right|\left|\Delta \widetilde{U}_{\varepsilon}\right| d x \\
& \left.+\int_{\mathbb{R}^{N}}\left|\Delta \widetilde{U}_{\varepsilon}\right|\left|\nabla \sigma_{\eta, y}\right|\left|\nabla \widetilde{U}_{\varepsilon}\right| d x+\int_{\mathbb{R}^{N}}\left|\Delta \widetilde{U}_{\varepsilon}\right|\left|\nabla \rho_{r}\right|\left|\nabla \widetilde{U}_{\varepsilon}\right| d x\right) .
\end{aligned}
$$

Since we have

$$
\nabla \widetilde{U}_{\varepsilon}=-C_{N}(N-4)\left(\frac{\varepsilon}{\varepsilon^{2}+|x|^{2}}\right)^{\frac{N-4}{2}} \frac{x}{\varepsilon^{2}+|x|^{2}}
$$

and

$$
\Delta \widetilde{U}_{\varepsilon}=C_{N}(N-4)\left(\frac{\varepsilon}{\varepsilon^{2}+|x|^{2}}\right)^{\frac{N-4}{2}}\left((N-2) \frac{|x|^{2}}{\left(\varepsilon^{2}+|x|^{2}\right)^{2}}-N \frac{1}{\varepsilon^{2}+|x|^{2}}\right)
$$

we can obtain the following inequalities:

$$
\begin{aligned}
& \left.\left|\int_{\mathbb{R}^{N}}\left(\sigma_{\eta, y}^{2} \rho_{r}^{2}-1\right)\right| \Delta \widetilde{U}_{\varepsilon}\right|^{2} d x \mid \\
& \quad \leq \int_{\mathbb{R}^{N}}\left|\sigma_{\eta, y}^{2} \rho_{r}^{2}-\sigma_{\eta, y}^{2}\right|\left|\Delta \widetilde{U}_{\varepsilon}\right|^{2} d x+\int_{\mathbb{R}^{N}}\left|\sigma_{\eta, y}^{2}-1\right|\left|\Delta \widetilde{U}_{\varepsilon}\right|^{2} d x \\
& \quad \leq \int_{|x| \geq r / 2}\left|\Delta \widetilde{U}_{\varepsilon}\right|^{2} d x+\int_{|x-y| \leq \eta}\left|\Delta \widetilde{U}_{\varepsilon}\right|^{2} d x \\
& \leq C \int_{r / 2}^{\infty} \frac{\varepsilon^{N-4}}{\left(\varepsilon^{2}+\varrho^{2}\right)^{N-4}}\left(\frac{\varrho^{4}}{\left(\varepsilon^{2}+\varrho^{2}\right)^{4}}+\frac{1}{\left(\varepsilon^{2}+\varrho^{2}\right)^{2}}\right) \varrho^{N-1} d \varrho \\
& \quad+C \int_{|x-y| \leq \eta}\left(\frac{\varepsilon}{\varepsilon^{2}}\right)^{N-4}\left(\frac{1}{\varepsilon^{2}}\right)^{2} d x \leq C\left(\frac{\varepsilon}{r}\right)^{N-4}+C\left(\frac{\eta}{\varepsilon}\right)^{N}, \\
& \int_{\mathbb{R}^{N}}\left|\Delta \sigma_{\eta, y}\right|^{2} \widetilde{U}_{\varepsilon}^{2} d x \leq \frac{C}{\eta^{4}} \int_{|x-y| \leq \eta}\left(\frac{\varepsilon}{\varepsilon^{2}}\right)^{N-4} d x \leq C\left(\frac{\eta}{\varepsilon}\right)^{N-4},
\end{aligned}
$$




$$
\begin{aligned}
& \int_{\mathbb{R}^{N}}\left|\Delta \rho_{r}\right|^{2} \widetilde{U}_{\varepsilon}^{2} d x \leq \frac{C}{r^{4}} \int_{r / 2 \leq|x| \leq r}\left(\frac{\varepsilon}{r^{2}}\right)^{N-4} d x \leq C\left(\frac{\varepsilon}{r}\right)^{N-4}, \\
& \int_{\mathbb{R}^{N}}\left|\nabla \sigma_{\eta, y}\right|^{2}\left|\nabla \widetilde{U}_{\varepsilon}\right|^{2} d x \leq \frac{C}{\eta^{2}} \int_{|x-y| \leq \eta}\left(\frac{\varepsilon}{\varepsilon^{2}}\right)^{N-4}\left(\frac{1}{\varepsilon}\right)^{2} d x \leq C\left(\frac{\eta}{\varepsilon}\right)^{N-2}, \\
& \int_{\mathbb{R}^{N}}\left|\nabla \rho_{r}\right|^{2}\left|\nabla \widetilde{U}_{\varepsilon}\right|^{2} d x \leq \frac{C}{r^{2}} \int_{r / 2 \leq|x| \leq r}\left(\frac{\varepsilon}{r^{2}}\right)^{N-4}\left(\frac{r}{r^{2}}\right)^{2} d x \leq C\left(\frac{\varepsilon}{r}\right)^{N-4}, \\
& \int_{\mathbb{R}^{N}} \widetilde{U}_{\varepsilon}\left|\Delta \sigma_{\eta, y}\right|\left|\Delta \widetilde{U}_{\varepsilon}\right| d x \leq \frac{C}{\eta^{2}} \int_{|x-y| \leq \eta}\left(\frac{\varepsilon}{\varepsilon^{2}}\right)^{\frac{N-4}{2}}\left(\frac{\varepsilon}{\varepsilon^{2}}\right)^{\frac{N-4}{2}} \frac{1}{\varepsilon^{2}} d x \leq C\left(\frac{\eta}{\varepsilon}\right)^{N-2}, \\
& \int_{\mathbb{R}^{N}} \widetilde{U}_{\varepsilon}\left|\Delta \rho_{r}\right|\left|\Delta \widetilde{U}_{\varepsilon}\right| d x \leq \frac{C}{r^{2}} \int_{r / 2 \leq|x| \leq r}\left(\frac{\varepsilon}{r^{2}}\right)^{\frac{N-4}{2}}\left(\frac{\varepsilon}{r^{2}}\right)^{\frac{N-4}{2}} \frac{r}{r^{2}} d x \leq C\left(\frac{\varepsilon}{r}\right)^{N-4}, \\
& \int_{\mathbb{R}^{N}} \widetilde{U}_{\varepsilon}\left|\nabla \sigma_{\eta, y}\right|\left|\nabla \rho_{r}\right|\left|\Delta \widetilde{U}_{\varepsilon}\right| d x \\
& \leq \frac{1}{2}\left(\int_{\mathbb{R}^{N}} \widetilde{U}_{\varepsilon}\left|\nabla \sigma_{\eta, y}\right|^{2}\left|\Delta \widetilde{U}_{\varepsilon}\right| d x+\int_{\mathbb{R}^{N}} \widetilde{U}_{\varepsilon}\left|\nabla \rho_{r}\right|^{2}\left|\Delta \widetilde{U}_{\varepsilon}\right| d x\right) \\
& \leq C\left(\frac{\eta}{\varepsilon}\right)^{N-2}+C\left(\frac{\varepsilon}{r}\right)^{N-4}, \\
& \int_{\mathbb{R}^{N}}\left|\Delta \widetilde{U}_{\varepsilon}\right|\left|\nabla \sigma_{\eta, y}\right|\left|\nabla \widetilde{U}_{\varepsilon}\right| d x \leq \frac{C}{\eta} \int_{|x-y| \leq \eta}\left(\frac{\varepsilon}{\varepsilon^{2}}\right)^{\frac{N-4}{2}} \frac{1}{\varepsilon^{2}}\left(\frac{\varepsilon}{\varepsilon^{2}}\right)^{\frac{N-4}{2}} \frac{1}{\varepsilon} d x \\
& \leq C\left(\frac{\eta}{\varepsilon}\right)^{N-1}, \\
& \int_{\mathbb{R}^{N}}\left|\Delta \widetilde{U}_{\varepsilon}\right|\left|\nabla \rho_{r}\right|\left|\nabla \widetilde{U}_{\varepsilon}\right| d x \leq \frac{C}{r} \int_{r / 2 \leq|x| \leq r}\left(\frac{\varepsilon}{r^{2}}\right)^{\frac{N-4}{2}} \frac{1}{r^{2}}\left(\frac{\varepsilon}{r^{2}}\right)^{\frac{N-4}{2}} \frac{r}{r^{2}} d x \\
& \leq C\left(\frac{\varepsilon}{r}\right)^{N-4}
\end{aligned}
$$

From the inequalities above, we can obtain (4.3). By

$$
\begin{aligned}
& \left.\left|\int_{\Omega}\right| \sigma_{\eta, y} \widetilde{U}_{\varepsilon}^{r}\right|^{2^{\sharp}} d x-\left.\widetilde{S}^{\frac{N}{4}}\left|\leq \int_{|x| \geq r / 2}\right| \widetilde{U}_{\varepsilon}\right|^{2^{\sharp}} d x+\int_{|x-y| \leq \eta}\left|\widetilde{U}_{\varepsilon}\right|^{2^{\sharp}} d x \\
& \quad \leq C \int_{r / 2}^{\infty}\left(\frac{\varepsilon}{\varrho^{2}}\right)^{N} \varrho^{N-1} d \varrho+C \int_{|x-y| \leq \eta}\left(\frac{\varepsilon}{\varepsilon^{2}}\right)^{N} d x \leq C\left(\frac{\varepsilon}{r}\right)^{N}+C\left(\frac{\eta}{\varepsilon}\right)^{N},
\end{aligned}
$$

we have (4.4). We also have

$$
\int_{\Omega}\left|\sigma_{\eta, y} \widetilde{U}_{\varepsilon}^{r}\right|^{2} d x \geq \frac{C}{\varepsilon^{N-4}} \int_{|x| \leq \varepsilon} \sigma_{\eta, y}^{2} d x \geq \frac{C}{\varepsilon^{N-4}}\left(\varepsilon^{N}-\eta^{N}\right) \geq C \varepsilon^{4} .
$$

Hence we have obtained the desired inequalities. 
Proof of Theorem 1.3. Let $\varepsilon_{r}=r^{\frac{N+4}{4}}$, and we define $\tilde{u}_{r}=\widetilde{U}_{\varepsilon_{r}}^{r}$. Then it is easy to see that $\tilde{u}_{r}$ is continuous in $\widetilde{H}$ with respect to $r \in\left(0, r_{2}\right]=\left(0, \frac{1}{6 m}\right]$. Let $\eta \in\left[0, r^{\frac{N+2}{2}}\right]$. Note that $N \geq 10$ and (4.2), by similar proof of Lemma 2.8, we show that

$$
\begin{gathered}
\sup _{t \geq 0} I\left(t \widetilde{\xi}_{\eta}(x-y) \tilde{u}_{r}(x)\right) \begin{cases}<\frac{2}{N} \widetilde{S}^{N / 4} & \text { if } r \in\left(0, r_{2}\right], \\
\leq \widetilde{S}_{m} & \text { if } r \in\left[\frac{r_{2}}{2}, r_{2}\right]\end{cases} \\
\widetilde{S}_{m}=\frac{2}{N} \widetilde{S}^{N / 4}-C_{7} m^{-(N+4)}, \\
\widetilde{S}_{m}+C_{5} m^{-\frac{N(N-4)}{4}}<\frac{2}{N} \widetilde{S}^{N / 4} .
\end{gathered}
$$

Now, let $\eta_{r}=r^{\frac{N+2}{2}}$. Following similar arguments of Lemma 2.9, Lemma 2.10 and Lemma 2.11, we get that $\beta_{n+N+2}<2^{4 / N} \widetilde{S}$. Therefore, by a similar argument as in the proof of Theorem 1.1, we can show Theorem 1.3.

Finally, we give a sketch of a proof of Theorem 1.4. Let $N \geq 8$, and $\widetilde{\lambda}_{n} \leq \lambda<\widetilde{\lambda}_{n+1}$ for some $n \geq 1$. By similar proofs of Lemma 3.1, we have the following crucial lemma.

Lemma 4.3. If $u$ is a nontrivial solution of (1.2) (respectively (1.3)), then

$$
\begin{gathered}
\|u\|_{2^{\sharp}}>\left(1-\frac{\lambda}{\widetilde{\lambda}_{n+1}}\right)^{\frac{N-4}{8}} \widetilde{S}^{\frac{N-4}{8}}, \\
\|u\|>\left(1-\frac{\lambda}{\widetilde{\lambda}_{n+1}}\right)^{\frac{N-4}{8}} \widetilde{S}^{\frac{N}{8}}, \\
\tilde{I}(u)>\frac{2}{N}\left(1-\frac{\lambda}{\widetilde{\lambda}_{n+1}}\right)^{N / 4} \widetilde{S}^{N / 4} .
\end{gathered}
$$

Let $\widetilde{K}:=\left\{u \in \widetilde{H}: u \neq 0, \tilde{I}^{\prime}(u)=0\right\}$. Define $\tilde{c}:=\inf _{u \in \widetilde{K}} \tilde{I}(u)$. Similar to Theorem 3.1, we have the following theorem.

Theorem 4.1. If problem (1.2)(respectively (1.3)) has a nontrivial solution $u$ with $\tilde{I}(u)<\frac{2}{N} \widetilde{S}^{N / 4}$, then there exists $u_{0} \in \widetilde{K}$, such that $\tilde{I}\left(u_{0}\right)=\tilde{c}$, i.e., $u_{0}$ is a ground state solution of problem (1.2) (respectively (1.3)).

Proof of Theorem 1.4. When $N=8,9, \lambda \neq \widetilde{\lambda}_{n}$ or $N \geq 10$, problem (1.2) (respectively (1.3)) has a nontrivial solution $u$ with $\tilde{I}(u)<\frac{2}{N} \widetilde{S}^{N / 4}[16]$. Therefore, by Lemma 4.3 and Theorem 4.1, problem (1.2) (respectively (1.3)) has a ground state solution $u$ with $\tilde{I}(u)>\frac{2}{N}\left(1-\frac{\lambda}{\widetilde{\lambda}_{n+1}}\right)^{N / 4} \widetilde{S}^{N / 4}$.

\section{References}

[1] Aubin, T.: Problemes isoperimetriques et espaces de Sobolev. J. Differ. Geom. 11, 573-598 (1976) 
[2] Atkinson, F.V., Brezis, H., Peletier, L.A.: Nodal solutions of elliptic equations with critical Sobolev exponents. J. Differ. Equ. 85, 151-170 (1990)

[3] Arioli, G., Gazzola, F., Grunau, H.C., Sassone, E.: The second bifurcation branch for radial solutions of the Brezis-Nirenberg problem in dimension four. Nonlinear Differ. Equ. Appl. 15, 69-90 (2008)

[4] Bernis, F., Grunau, H.C.: Critical exponents and multiple critical dimensions for polyharmonic operators. J. Differ. Equ. 117, 469-486 (1995)

[5] Brezis, H., Lieb, E.: A relation between pointwise convergence of functions and convergence of functionals. Proc. Am. Math. Soc. 88, 486-490 (1983)

[6] Brezis, H., Nirenberg, L.: Positive solutions of nonlinear elliptic equations involving critical Sobolev exponents. Commun. Pure Appl. Math. 36, 437-477 (1983)

[7] Capozzi, A., Fortunato, D., Palmieri, G.: An existence result for nonlinear elliptic problems involving critical Sobolev exponent. Ann. Inst. H. Poincare Analyse Nonlinear 2, 463-470 (1985)

[8] Cerami, G., Fortunato, D., Struwe, M.: Bifurcation and multiplicity results for nonlinear elliptic problems involving critical Sobolev exponents. Ann. Inst. H. Poincare Analyse Nonlinear 1, 341-350 (1984)

[9] Cao, D., Han, P.: Solutions for semilinear elliptic equations with critical exponents and Hardy potential. J. Differ. Equ. 205, 521-537 (2004)

[10] Cerami, G., Solimini, S., Struwe, M.: Some existence results for superlinear elliptic boundary value problems involving critical exponents. J. Funct. Anal. 69, 289-306 (1986)

[11] Clapp, M., Weth, T.: Multiple solutions for the Brezis-Nirenberg problem. Adv. Differ. Equ. 10, 463-480 (2005)

[12] Devillanova, G., Solimini, S.: Concentration estimates and multiple solutions to elliptic problems at critical growth. Adv. Differ. Equ. 7, 1257-1280 (2002)

[13] Edmunds, D.E., Fortunato, D., Jannelli, E.: Critical exponents, critical dimensions and the biharmonic operator. Arch. Rational Mech. Anal. 112, 269-289 (1990)

[14] Ferrero, A., Gazzola, F.: Existence of solutions for singular critical growth semilinear elliptic equations. J. Differ. Equ. 177, 494-522 (2001)

[15] Fortunato, D., Jannelli, E.: Infinitely many solutions for some nonlinear elliptic problems in symmetrical domains. Proc. R. Soc. Edinburgh 105A, 205-213 (1987)

[16] Gazzola, F.: Critical growth problems for polyharmonic operators. Proc. R. Soc. Edinburgh A 128, 251-263 (1998)

[17] Gazzola, F., Grunau, H.C., Squassina, M.: Existence and nonexistence results for critical growth biharmonic elliptic equations. Calc. Var. 18, 117-143 (2003) 
[18] Grunau, H.C., Sweers, M.: Positivity for equations involving polyharmonic operators with Dirichlet boundary conditions. Math. Ann. 307, 589-626 (1997)

[19] Pankov, A.: Periodic nonlinear Schrodinger equation with application to photonic crystals. Milan J. Math 73, 259-287 (2005)

[20] Pucci, P., Serrin, J.: Critical exponents and critical dimensions for polyharmonic operators. J. Math. Pure Appl. 69, 55-83 (1990)

[21] Rabinowitz, P.H.: Minimax methods in critical point theory with application to differential equation. In: CBMS, vol. 65. AMS, Providence (1986)

[22] Szulkin, A., Weth, T., Willem, M.: Ground state solutions for a semilinear problem with critical exponent. Differ. Int. Equ. 22, 913-926 (2009)

[23] Schechter, M., Zou, W.: On the Brezis-Nirenberg problem. Arch. Rational Mech. Anal. 197, 337-356 (2010)

[24] Struwe, M.: A global compactness result for elliptic boundary value problems involving limiting nonlinearities. Math. Z. 187, 511-517 (1984)

[25] Struwe, M: Variational methods. Applications to Nonlinear Partial Differential Equations and Hamiltonian Systems. Springer, Berlin (1996)

[26] Solimini, S.: A note on compactness-type properties with respect to Lorenz norms of bounded subsets of a Sobolev spaces. Ann. Inst. H. Poincare Analyse Nonlinear 12, 319-337 (1995)

[27] Talenti, G.: Best constant in Sobolev inequality. Ann. Mat. Pure Appl. 110, 352-372 (1976)

[28] Van der Vorst, R.C.A.M.: Best constant for the embedding of the space $H^{2} \cap$ $H_{0}^{1}(\Omega)$ into $L^{2 N /(N-4)}(\Omega)$,. Differ. Int. Equ. 6, 259-276 (1993)

[29] Van der Vorst, R.C.A.M.: Fourth-order elliptic equations with critical growth. C. R. Acad. Sci. Paris Sér. I Math. 320, 295-299 (1995)

[30] Yamabe, H.: On the deformation of Riemannian structures on compact manifold. Osaka Math. J. 12, 21-37 (1960)

[31] Zhang, D.: On multiple solutions of $\Delta u+\lambda u+|u|^{\frac{4}{n-2}} u=0$,. Nonlinear Anal. TMA 13, 353-372 (1989)

Z. Chen and W. Zou

Department of Mathematical Sciences

Tsinghua University

Beijing 100084

China

e-mail:wzou@math.tsinghua.edu.cn

Z. Chen

e-mail: chenzhijie1987@sina.com 
N. Shioji

Department of Mathematics

Faculty of Engineering

Yokohama National University

Tokiwadai, Hodogaya-ku

Yokohama 240-8501

Japan

e-mail: shioji@math.sci.ynu.ac.jp

Received: 16 October 2010.

Accepted: 17 June 2011. 